\title{
Existence of Hypersurfaces with Prescribed Mean Curvature in Riemannian Manifolds
}

\author{
Frank Duzaar $\mathscr{E}$ Klaus Steffen
}

0. Introduction. In this work we treat the Plateau problem for hypersurfaces of prescribed mean curvature in a Riemannian manifold: Given a complete oriented Riemannian manifold $N$ of dimension $n+1$, a function $H: N \rightarrow \mathbb{R}$, and a surface $B$ of codimension 2 in $N$ we want to find an oriented hypersurface $T$ with boundary $B$ in $N$ such that $T$, at each point $x$ of its support, has the mean curvature $H(x)$ specified there in advance. We fix a reference hypersurface $T_{0}$ with boundary $\partial T_{0}=B$ and we look for a hypersurface of prescribed mean curvature in the homology class of $T_{0}$, i.e. we require additionally that $T-T_{0}$ is the boundary of some $n+1$ chain in $N$. The classical Plateau problem is to find a surface with given boundary which is of least area or, more generally, stationary for the area functional; this corresponds to a vanishing prescribed mean curvature function $H \equiv 0$.

In the setting of 2 dimensional parametric surfaces in Euclidean space $\mathbb{R}^{3}$ the Plateau problem with prescribed mean curvature was treated for the first time by Heinz [He1]. A further breakthrough was achieved by Hildebrandt [Hi1], [Hi2] who proved, first for constant $H$ and then also for variable $H$, existence of a parametric surface of disk type with given boundary in the unit ball of $\mathbb{R}^{3}$ and with prescribed mean curvature $H$ under the optimal condition $|H| \leq 1$. Further contributions giving sufficient conditions for $H$ of a different nature were made by Wente [We], Gulliver and Spruck [GS1], [GS2], and the second author [St1], [St2]. For an account of some of the results on the Plateau problem for parametric surfaces of prescribed mean curvature in $\mathbb{R}^{3}$ we refer also to Struwe [Str].

More recently, the Plateau problem with prescribed mean curvature was studied in the context of integer multiplicity rectifiable $n$ currents $T$ on $\mathbb{R}^{n+1}$ by Duzaar and Fuchs [DF1], [DF2] and the first author [Du]. They succeeded to carry over to this setting many of the results from the parametric theory. The corresponding regularity theory was developed by the authors [Du], [DS1], [DS2]. The (energy minimizing) solutions to the Plateau problem with prescribed mean curvature exhibit the same degree of regularity as the absolutely area minimizing codimension 1 currents, i.e. they are classical hypersurfaces 
smooth up to the boundary for $n \leq 6$, and for $n \geq 7$ the possible closed singular set is of codimension at least 7 and disjoint from the support of the boundary. (Here, the given boundary and the prescribed mean curvature are assumed to be sufficiently smooth.)

In the present paper we study the Plateau problem with prescribed mean curvature for integer multiplicity rectifiable currents of codimension 1 in a Riemannian manifold $N$ (satisfying the assumptions described in Section 1 below). Our interest in this problem is motivated by the fact that new phenomena arise when one passes from Euclidean space $\mathbb{R}^{n+1}$ to a Riemannian manifold $N$. Namely, in contrast with the Euclidean situation, it may then happen that no hypersurface $T$ with $\partial T=\partial T_{0}$ (and homologous to $T_{0}$ ) exists in $N$ which has constant mean curvature different from zero. For example, this situation occurs when $T_{0}$ represents a great half sphere in the standard sphere $N=S^{n+1}$ and also when $T_{0}$ represents the cross section $M \times\{0\}$ in a cylinder $N=M \times \mathbb{R}$. Non-existence results of this type for 2 dimensional parametric surfaces, which are valid also for currents, were proved by Gulliver [Gu2], [Gu3]. In Section 1 below (Remark 1.4) we briefly describe his reasoning, in the simplest case.

Hildebrandt and Kaul [HiK] and Gulliver [Gu1] have also studied the Plateau problem for parametric surfaces of prescribed mean curvature in a Riemannian 3 manifold. Their methods can be adapted to prove analogous results for $n$ currents in a Riemannian manifold $N$ of dimension $n+1$. However, we can go beyond this. For example, the results of $[\mathrm{HiK}],[\mathrm{Gu1}]$ are limited to boundaries contained in a normal star-shaped domain of $N$, while we are able to treat boundaries which can be spanned by a reference current $T_{0}$ of sufficiently small $n$ area not assuming any further condition which restricts the shape of the boundary. Our results are quantitatively optimal with respect to the hypotheses on $T_{0}$ which ensure, for all sufficiently small functions $H$, the existence of an $n$ current $T$ with mean curvature $H$ in the homology class of $T_{0}$.

With some extra work the methods used in this paper can also be applied to improve the known results and to prove some new results about the existence of parametric surfaces of prescribed mean curvature in Riemannian 3 manifolds. We will present this in a separate paper [DS4].

We now describe the content of this paper in more detail. In Section 1 we introduce the notation and terminology: we define the notion of mean curvature for $n$ currents $T$ in an oriented $n+1$ manifold $N$ (following Allard [Al]. Warning: we also follow Allard's convention that the mean curvature is the sum-not the arithmetic mean - of the principal curvatures), and we establish the variational character for the equation of prescribed mean curvature. We may impose a constraint spt $T \subset \bar{G}$ on the support of $T$, the exterior $N \backslash \bar{G}$ of the domain $G$ in $N$ being considered as an obstacle for $T$. In Lemma 1.5 we prove that $T$ has mean curvature $H$, if $T$ satisfies the relevant variational inequality associated with the prescribed function $H$ and the constraint $\operatorname{spt} T \subset \bar{G}$ and if $H_{G}$, the (inward) mean boundary curvature of $\partial G$, satisfies $H_{G} \geq|H|$ along $\partial G$. For 2 -dimensional parametric surfaces such a result, requiring only the variational inequality and no minimizing property, has been proved by Hildebrandt [Hi3], [Hi4] (see also [GS2], [DF1], [DS3]). 
The variational functional $\mathbf{E}_{H}$ associated with the equation of prescribed mean curvature $H$ has the form

$$
\mathbf{E}_{H}(T)=\mathbf{M}(T)+\mathbf{V}_{H}\left(T, T_{0}\right),
$$

where $\mathbf{M}(T)$ denotes the mass ( $n$ area) of $T$ and $\mathbf{V}_{H}\left(T, T_{0}\right)$ the volume, with respect to the weight function $H$, of the $n+1$ current $Q$ bounded by $T-T_{0}$. In order to minimize $\mathbf{E}_{H}$ on suitable subsets of the homology class of $T_{0}$, one must be able to dominate the volume term in $(0.1)$ by the mass term. Therefore, isoperimetric inequalities in the manifold $N$ are fundamental for our existence results. We review in Section 2 such isoperimetric inequalities, of the "linear" type

of the "nonlinear" type

$$
|A| \leq c|\partial A|
$$

$$
|A| \leq \gamma|\partial A|^{(n+1) / n},
$$

and of more general forms. (Here $|A|$ and $|\partial A|$ denote the Riemannian volume and boundary $n$ area of sets $A \subset N$ with suitable structure.) Since $Q$ above is, in general, not represented by a characteristic function but rather by an integer valued function of bounded variation, we also discuss the implications of isoperimetric inequalities for functions of bounded variation on $N$. These implications can be expressed by certain Sobolev type inequalities.

In Section 2 we discuss isoperimetric inequalities in Riemannian manifolds. Much of the material we present there is known. However, since isoperimetric inequalities are important for the results of this paper and we could not always find them in the form needed here, we have chosen to give an exposition of the ideas and to indicate proofs wherever this seemed possible with reasonable effort. From the results in Section 2 that are new or could not be traced in the literature we mention Proposition 2.2, which relates Morrey's condition of homogeneous regularity of $N$ (a uniformity condition at infinity) to isoperimetric inequalities on $N$, and Proposition 2.9, which establishes an isoperimetric inequality $(0.2)$ for sets $A \subset G$ if the (inward) mean curvature $H_{G}(x)$ of the local parallel surface to $\partial G$ through $x$, at any point $x \in G$ where this is defined, is not smaller than $c^{-1}$. We also mention Proposition 2.11 on isoperimetric inequalities in cylinders $N=M \times \mathbb{R}$, which we have not found in the literature.

In Section 3 we treat our existence problem in a non-closed ambient space $\bar{G}$, i.e. either $G=N$ is complete, non-compact, with empty boundary, or $G$ is a smooth proper subdomain of the complete manifold $N$. In this case there is a unique $n+1$ current $Q$ with spt $Q \subset \bar{G}$ and boundary $\partial Q=T-T_{0}$, whenever $T, T_{0}$ are two given homologous integer multiplicity rectifiable $n$ currents in $N$ with support in $\bar{G}$. Therefore, the volume $\mathbf{V}_{H}\left(T, T_{0}\right)$ is uniquely defined by integrating $H$ over $Q$, and it can be dominated by the mass $\mathbf{M}\left(T-T_{0}\right)$ in various ways using isoperimetric inequalities (cf. Proposition 3.4). In Theorem 3.2 we present a general result on the solution of the Plateau problem with prescribed mean curvature $H$ that is based on the minimization of the energy $\mathbf{E}_{H}$ from (0.1) on certain classes of $n$ currents in $N$. If an isoperimetric inequality (0.2) holds with a constant $c<\infty$ for all $A \subset N$, then we get existence for 
all reference currents $T_{0}$ and all functions $H$ with $\sup _{N}|H|<c^{-1}$ (Theorem 3.6 (i)). This can be applied, in particular, in simply connected manifolds $N$ with sectional curvatures $\leq K<0$; then $c^{-1} \geq n \sqrt{-K}$ holds (Corollary 3.7). The anologous conclusions are true with a constraint spt $T \subset \bar{G}$, provided we have also $H_{G} \geq|H|$ along $\partial G$.

In Theorem 3.9 we consider the case where we have isoperimetric inequalities of both types (0.2), (0.3) for all $A \subset G$ with finite constants $c$ and $\gamma$ and we prove various sufficient criteria for existence, e.g. $H_{G} \geq|H|$ along $G$ and

$$
\int_{\{x \in G: c|H(x)|>n /(n+1)\}}|H(x)|^{n+1} d x<\gamma^{-n} .
$$

In Theorem 3.10 we specialize $G$ to a geodesically star-shaped normal neighborhood of a point $a$ in a manifold $N$ with sectional curvatures $\leq K$. We assume spt $T_{0} \subset \bar{G}$ and $H_{G} \geq|H|$ along $\partial G$ and we denote by $\varrho(x)$ the length of the geodesic segment in $G$ connecting $x$ to $a$. We then prove existence of a solution to our Plateau problem with data $T_{0}$ and $H$ in $\bar{G}$ if

$$
\sup _{G} \varrho \leq r \quad \text { and } \quad \sup _{G}|H|<b(r, K)
$$

where $b(r, K)$ is the ratio of the boundary area to the volume of a ball with radius $r$ in the $n+1$ dimensional standard space with constant sectional curvature $K$. (We also assume $\sqrt{K} r<\frac{1}{2} \pi$ if $K>0$.) Results of this type can be derived from $[\mathrm{HiK}],[\mathrm{Gu} 1]$ (adapting the reasoning there to the present setting), with stronger assumptions on $H$ and $G$, however. For example, the following condition was imposed in [Gu1] if $G$ is a normal ball of radius $r$ :

$$
\sup _{G}|H|<\left\{\begin{array}{cl}
n \sqrt{|K|} \operatorname{coth} \sqrt{|K|} r & \text { if } K<0, \\
n r^{-1} & \text { if } K=0, \\
n \sqrt{K} \cot \sqrt{K} r & \text { if } K>0 .
\end{array}\right.
$$

Our result gives improvements in several respects; we admit more general domains, the constant $b(r, K)$ in $(0.5)$ is larger than the right-hand side of $(0.6)$ (by a factor $(n+1) / n$, if $K=0$; cf. also [GS2]), and the barrier condition $H_{G}>|H|$ on $\partial G$ is implied by $(0.6)$ for a normal geodesic ball $G$ of radius $r$. Moreover, we obtain also existence under conditions which do not imply a bound for $\sup _{G}|H|$ at all, e.g. integral conditions of type (0.4) or the condition

$(0.7)|H(x)| \leq\left\{\begin{array}{cc}c n \sqrt{|K|} \operatorname{coth} \sqrt{|K|} \varrho(x) & \text { if } K<0, \\ c \varrho(x)^{-1} & \text { if } K=0, \\ c n \sqrt{K} \cot \sqrt{K} \varrho(x) & \text { if } K>0,\end{array}\right\} \quad$ for $x \in G \backslash\{a\}$

with a constant $c<1$. For a normal geodesic ball $G$ of radius $r(0.7)$ is an obvious improvement over (0.6).

At the end of Section 3 we treat the case where an isoperimetric inequality (0.2) with a finite constant $c$ is only valid for sets $A \subset G$ satisfying a certain 
restriction $|\partial A| \leq s<\infty$. We prove in Theorem 3.11 that a current $T$ with prescribed mean curvature $H$ and $\operatorname{spt} T \subset \bar{G}$ exists in the homology class of $T_{0}$ if $\operatorname{spt} T_{0} \subset \bar{G}, H_{G} \geq|H|$ along $\partial G$, and

$$
\sup _{G}|H|<\frac{s-2 \mathbf{M}\left(T_{0}\right)}{s c} .
$$

This is an analog of a result of Wente [We] and its generalizations in [St2], [Du]. It implies, in particular, that a given boundary in $N$ can be spanned by a hypersurface of large prescribed (e.g. constant) mean curvature as soon as it can be spanned by a hypersurface of small area (Corollary 3.13). In the case of a cylinder $N=M \times \mathbb{R}(0.8)$ can be fulfilled with functions $H \not \equiv 0$ whenever $\mathbf{M}\left(T_{0}\right)$ is smaller than the volume of $M$, while for $T_{0}$ representing the cross section $M \times\{0\}$ in $N$ and for constant $H \not \equiv 0$ no current of prescribed mean curvature $H$ exists in the homology class of $T_{0}$ (Example 3.12). Thus, the condition imposed on $T_{0}$ by inequality $(0.8)$ is optimal in quantitative respect.

In the final Section 4 we treat closed (i.e. compact without boundary) ambient manifolds $N$. The problem here is that the $n+1$ current $Q$ with boundary $\partial Q=T-T_{0}$ is determined only up to an integer multiple of $N$ and hence the volume enclosed by $T$ and $T_{0}$ is not well defined. There are two possibilities to circumvent this. First, the problem simply disappears if we impose the condition $\int_{N} H(x) d x=0$ on the prescribed mean curvature function, since $H$ is used as a weight function in the definition of the volume term $\mathbf{V}_{H}\left(T, T_{0}\right)$ in (0.1). Indeed, with this assumption all the results from Section 3 can be carried over to closed manifolds without difficulties (Theorem 4.1, Corollary 4.2). However, the condition of vanishing mean value excludes constant $H \not \equiv 0$ which we find undesirable. We therefore have chosen a second route to avoid the indeterminacy of the volume, namely, we work in classes of $n$ currents $T$ such that $T-T_{0}=\partial Q$ holds for some $n+1$ current with mass $\mathbf{M}(Q)<\frac{1}{2}|N|$. Such $Q$ is then unique and can be used to define $\mathbf{V}_{H}\left(T, T_{0}\right)$ without ambiguity. Following this approach we prove in Theorem 4.3 existence of a current with prescibed mean curvature $H$ homologous to $T_{0}$ in $N$ if the isoperimetric inequality (0.2) is valid with the constant $c$ for all $A \subset N$ with $|\partial A| \leq s$, if $c s<\frac{1}{2}|N|$ holds and if the inequality (0.8) is satisfied (with $G=N$ ). In the case $N=S^{n+1}$ the latter condition can be fulfilled with functions $H \not \equiv 0$ whenever $\mathbf{M}\left(T_{0}\right)$ is smaller than the $n$ area of a great half sphere in $S^{n+1}$, while for $T_{0}$ representing such a half sphere and for $H$ constant $\not \equiv 0$ we have non-existence (Example 4.4).

As our last result we prove that a condition weaker than (0.8), but still sufficient for the existence of a current with prescribed mean curvature $H$ in the homology class of $T_{0}$, is satisfied for $\sup _{N}|H|$ sufficiently small (but positive) whenever $T_{0}$ is uniquely mass minimizing in its homology class. (Instead of this uniqueness it suffices actually that no second mass minimizer bounds, together with $T_{0}$, a current of mass $\frac{1}{2}|N|$.) For complete non-closed manifolds which are homogeneously regular we prove an analogous statement in Theorem 4.7. It is interesting to contrast these results with the examples of non-existence mentioned previously, where indeed a large 1-parameter family of mass minimizers is contained in the homology class of $T_{0}$. 
1. Currents of prescribed mean curvature. We work in a smooth (class $C^{3}$ ), connected, complete, and oriented Riemannian manifold $N$ of dimension $n+1 \geq 2$. The Riemannian volume form on $N$ is denoted $\Omega$, the Riemannian measure on $N$ by $\mathcal{H}^{n+1}$ (Hausdorff measure). For tangent spaces of $N$ we use the notation $\operatorname{Tan}_{x} N$. We will assume that $N$ has no boundary but later consider also smooth domains $G \subset N$ in $N$ with $\partial G \neq \varnothing$ as ambient space for our currents.

Surfaces in $N$ will be modelled by locally rectifiable integer multiplicity currents in $N$ of finite mass. Such currents $T$, of dimension $m \in\{0,1, \ldots, n+1\}$, can be represented by an $\left(\mathcal{H}^{m}, m\right)$ rectifiable and $\mathcal{H}^{m}$ measurable supporting set $M_{T} \subset N$, an $\mathcal{H}^{m}$ summable multiplicity function $\vartheta_{T}: M_{T} \rightarrow \mathbb{N}$, and an $\mathcal{H}^{m}$ measurable orientation $\vec{T}: M_{T} \rightarrow \bigwedge_{m} \operatorname{Tan} N$, i.e. $\vec{T}$ is the exterior product of an orthonormal basis in the $m$ dimensional measure theoretic tangent space $\operatorname{Tan}\left(\mathcal{H}^{m}\left\llcorner M_{T}, x\right)\right.$ of $M_{T}$ which exists at $\mathcal{H}^{m}$ almost all points $x \in M_{T}$. The orthogonal projection in $\operatorname{Tan}_{x} N$ onto $\operatorname{Tan}\left(\mathcal{H}^{m} L M_{T}, x\right)$ is then denoted by $P_{\vec{T}}(x) \in \operatorname{Hom}\left(\operatorname{Tan}_{x} N, \operatorname{Tan}_{x} N\right)$. We set $\vartheta_{T} \equiv 0, \vec{T} \equiv 0$ outside $M_{T}$ and denote by $\|T\|=\mathcal{H}^{m}\left\llcorner\vartheta_{T}\right.$ the measure associated with $T$ and by $\mathbf{M}(T)=\|T\|(N)=$ $\int \vartheta_{T} d \mathcal{H}^{m}<\infty$ the mass of $T$.

By definition, an $m$ current in $N$ is a (continuous) linear functional on the space of compactly supported smooth differential $m$ forms on $N$. In terms of the quantities $\|T\|, \vec{T}$, the pairing of currents and differential forms is given by $\langle T, \alpha\rangle=\int\langle\alpha, \vec{T}\rangle d\|T\|$ and is defined whenever $\alpha$ is a bounded Baire form of degree $m$ on $N$. The boundary current $\partial T$ is then defined by taking formally the dual of the exterior derivative, i.e. $\langle\partial T, \beta\rangle=\langle T, d \beta\rangle$ for compactly supported smooth $m-1$ forms $\beta$ on $N$.

By $\mathcal{R}_{m}(N)$ we denote the class of locally rectifiable integer multiplicity $m$ currents of finite mass in $N$ as described above. With the addition defined for linear functionals on $m$ forms, $\mathcal{R}_{m}(N)$ is then an abelian group. Using an isometric embedding $J: N \rightarrow \mathbb{R}^{n+1+p}$ with closed image, we may identify $\mathcal{R}_{m}(N)$ with the set of currents in $\mathcal{R}_{m}\left(\mathbb{R}^{n+1+p}\right)$ having support in $N$. Every oriented $m$ dimensional submanifold $M$ (of multiplicity 1 ) with locally finite $m$ volume determines a locally rectifiable $m$ current $\llbracket M \rrbracket$ of multiplicity 1 in $N$ given by integration of compactly supported $m$ forms over $M$; if the volume $\mathcal{H}^{m}(M)$ is finite, then $\llbracket M \rrbracket \in \mathcal{R}_{m}(N)$. (In the case $M=[s, t] \subset \mathbb{R}$ we write $\llbracket s, t \rrbracket$ for the 1 current which corresponds to the integration of 1 forms over the interval $[s, t]$.)

The structure of top dimensional currents $Q \in \mathcal{R}_{n+1}(N)$ is simple: We may interprete $Q$ as a set with integer multiplicities in $N$ described by the integer valued function $\zeta_{Q}=\langle\Omega, \vec{Q}\rangle \vartheta_{Q}$ on $N$. For the currents $T \in \mathcal{R}_{n}(N)$ of codimension 1 (which we will take to represent hypersurfaces in $N$ ), we can use the unit normal field $* \vec{T}$ to describe their orientation $\vec{T}$. Here $*: \bigwedge_{n} \operatorname{Tan}_{x} N \rightarrow$ $\operatorname{Tan}_{x} N$ is the star isomorphism defined by $\tau \cdot(* \zeta)=\langle\Omega(x), \tau \wedge \zeta\rangle$ for $x \in N$, $\tau \in \operatorname{Tan}_{x} N$ and $\zeta \in \bigwedge_{n} \operatorname{Tan}_{x} N$. Then $* \overrightarrow{\llbracket G \rrbracket}$ is the exterior unit normal field of $G$ whenever $G$ is a smooth domain in $N$ with orientation inherited from $N$. (We warn the reader that different sign conventions with regard to the star operator 
are common and have been used by ourselves in previous work.)

For the general theory of currents we refer to [Fe], [Si]. Our notation related to currents and not explained above is consistent with these references. We will freely use concepts and results for currents in the manifold $N$ whenever their adaptation from the Euclidean situation to the Riemannian case is straightforward and does not present particular difficulties.

1.1. Definition. Given a continuous function $H: N \rightarrow \mathbb{R}$ and $T \in \mathcal{R}_{n}(N)$, we say that $T$ is a current of (prescribed) mean curvature $H$ in $N$ if

$$
\int P_{\vec{T}} \cdot D X d\|T\|+\int H(* \vec{T} \cdot X) d\|T\|=0
$$

holds for every $C^{1}$ vector field $X$ on $N$ with compact support in $N \backslash$ spt $\partial T$.

Here $D X(x) \in \operatorname{Hom}\left(\operatorname{Tan}_{x} N, \operatorname{Tan}_{x} N\right)$ denotes the covariant differential of $X$ at $x \in N$ and $P_{\vec{T}}(x) \cdot D X(x)$ its inner product with the projection $P_{\vec{T}}(x)$, which is defined in terms of the natural inner product on $\operatorname{Hom}\left(\operatorname{Tan}_{x} N, \operatorname{Tan}_{x} N\right)$ induced by the Riemannian metric on $N$.

If $T=\llbracket M \rrbracket$ is represented by a smooth oriented hypersurface $M \subset N$ with orienting unit normal vectorfield $\nu=* \vec{T}$ and $\tau_{1}, \ldots, \tau_{n}$ is a local orthonormal frame on $M$, then we compute, using $P_{\vec{T}}=\mathrm{id}-(\nu \cdot) \otimes \nu$ :

$$
\begin{aligned}
P_{\vec{T}} \cdot D X & =\sum_{i=1}^{n} \tau_{i} \cdot P_{\vec{T}} D_{\tau_{i}} X=\sum_{i=1}^{n} \tau_{i} \cdot\left[D_{\tau_{i}}\left(P_{\vec{T}} X\right)-\left(D_{\tau_{i}} P_{\vec{T}}\right) X\right] \\
& =\sum_{i=1}^{n} \tau_{i} \cdot D_{\tau_{i}}\left(P_{\vec{T}} X\right)+\sum_{i=1}^{n} \tau_{i} \cdot\left[\left(D_{\tau_{i}} \nu \cdot X\right) \nu+(\nu \cdot X) D_{\tau_{i}} \nu\right] \\
& =\operatorname{div}_{M}\left(P_{\vec{T}} X\right)+\sum_{i=1}^{n}\left(\tau_{i} \cdot D_{\tau_{i}} \nu\right)(\nu \cdot X)=\operatorname{div}_{M}\left(P_{\vec{T}} X\right)-H_{M}(\nu \cdot X),
\end{aligned}
$$

where $H_{M}:=-\operatorname{trace}_{M} D \nu$ is the mean curvature of $M$ with respect to the ambient manifold $N$ and the normal field $\nu$. Integrating over $M$, we see that (1.1) for all $X$ is equivalent to the equation $H_{M}=H$ on $M \backslash \partial M$, hence Definition 1.1 states that, in the weak sense, $T$ has mean curvature $H$ and mean curvature vector $H(* \vec{T})$ on spt $T \backslash$ spt $\partial T$. (Our convention on the mean curvature here is that it is defined as the sum of the principal curvatures. Thus, the standard sphere $S^{n}$, oriented by the interior unit normal field has constant mean curvature $n$ in $\mathbb{R}^{n+1}$. This avoids a dimension factor $n$ in many of the subsequent formulas.)

1.2. Remarks. (1) When the vector field $X$ is orthogonal to $T$, i.e. $P_{\vec{T}} X \equiv$ 0 , then Equation (1.1) for a current $T$ of mean curvature $H$ is valid for bounded $X$ and $D X$ without the requirement that $X$ have compact support in $N \backslash$ spt $\partial T$. To see this, we consider a cut-off function $\eta$ with compact support in $N \backslash$ spt $\partial T$, 
replace $X$ by $\eta X$ in (1.1), note that $D(\eta X)=d \eta \otimes X+\eta D X$ and $P_{\vec{T}} \cdot(d \eta \otimes X)=$ 0 for $X$ orthogonal to $T$, and finally let $\eta$ approximate the characteristic function of $N \backslash$ spt $\partial T$. (To obtain (1.1) in the limit we must assume $\|T\|(\operatorname{spt} \partial T)=0$ or we replace the domain $N$ of integration in (1.1) by $N \backslash \operatorname{spt} \partial T$.)

(2) Using an isometric embedding $J: N \rightarrow \mathbb{R}^{n+1+p}$, we can compute the (generalized) mean curvature vector of $T$ from Definition 1.1 with respect to the ambient space $\mathbb{R}^{n+1+p}$ as follows. We choose a smooth local orthonormal frame $\nu_{1}, \ldots, \nu_{p}$ of normal vectors to $N$ and a local frame $\tau_{0}, \ldots, \tau_{n}$ of tangent vectors to $N$ such that $\vec{T}=\tau_{1} \wedge \ldots \wedge \tau_{n}$ holds $\|T\|$ almost everywhere. For $g \in C^{1}\left(\mathbb{R}^{n+1+p}, \mathbb{R}^{n+1+p}\right)$ with compact support in $\mathbb{R}^{n+1+p} \backslash$ spt $\partial T$, we then have, $\|T\|$ almost everywhere on $N$,

$$
\begin{aligned}
P_{\overrightarrow{J_{\#}}} \cdot D g & =\sum_{i=1}^{n} \tau_{i} \cdot\left[\partial_{\tau_{i}}\left(P_{N} g\right)+\partial_{\tau_{i}} \sum_{l=1}^{p}\left(\nu_{l} \cdot g\right) \nu_{l}\right] \\
& =P_{\vec{T}} \cdot D\left(\left.P_{N} g\right|_{N}\right)+\sum_{i=1}^{n} \sum_{l=1}^{p}\left(\tau_{i} \cdot \partial_{\tau_{i}} \nu_{l}\right)\left(\nu_{l} \cdot g\right) \\
& =P_{\vec{T}} \cdot D\left(\left.P_{N} g\right|_{N}\right)-g \cdot \operatorname{trace}_{\vec{T}} A_{N}
\end{aligned}
$$

where $P_{N}$ is the field of orthogonal projections $P_{N}(x): \mathbb{R}^{n+1+p} \rightarrow \operatorname{Tan}_{x} N$ and $A_{N}$ is the second fundamental form of $N$ with values in the normal bundle of $N$; the notation trace $\vec{T}$ refers to taking the trace of this form on the subspace of $\operatorname{Tan}_{x} N$ associated with $\vec{T}(x)$, for $\|T\|$ almost all $x$, with respect to the Riemannian metric of $N$ (resp. the canonical inner product in $\mathbb{R}^{n+1+p}$ ). Comparing with (1.1), we see that the current $J_{\#} T$ in $\mathbb{R}^{n+1+p}$ has mean curvature vector field

$$
H(* \vec{T})+\operatorname{trace}_{\vec{T}} A_{N}
$$

in the weak sense. Since this is locally bounded, we can apply to $T$ all the results valid for currents (or varifolds) of bounded mean curvature in Euclidean spaces, in particular the monotonicity formula (see [Al], [Si], [DS2]).

Equation (1.1) has a variational structure, and we shall use this to solve it for a prescribed mean curvature function $H$ by minimizing the corresponding variational integral in suitable subclasses of $\mathcal{R}_{n}(N)$. A convenient way to describe this variational structure is as follows:

1.3. Lemma. $T \in \mathcal{R}_{n}(N)$ has mean curvature $H$ on $N$ iff

$$
\left.\frac{d}{d t}\right|_{0}\left[\mathbf{M}\left(h_{t \#} T\right)+\left\langle h_{\#}(\llbracket 0, t \rrbracket \times T), H \Omega\right\rangle\right]=0
$$

holds for all deformations $h:[0,1] \times N \rightarrow N$ of $\operatorname{id}_{N}=h_{0}$ with $h$, $\partial h / \partial t$ of class $C^{1}$ and compact support $S$ in $N \backslash$ spt $\partial T$ (i.e. $h_{t}(x)=x$ for $x \in N \backslash S$ and $0 \leq t \leq 1$, where $\left.h_{t}(x)=h(t, x)\right)$. 
Proof. We have, for $t>0$ sufficiently small,

$$
\begin{aligned}
& \mathbf{M}\left(h_{t \#} T\right)=\int\left|\left(\bigwedge_{n} d h_{t}\right) \vec{T}\right| d\|T\|, \\
& \left\langle h_{\#}(\llbracket 0, t \rrbracket \times T), H \Omega\right\rangle=\int_{0}^{t} \int H\left\langle\Omega, \frac{\partial h}{\partial s} \wedge\left(\bigwedge_{n} d h_{s}\right) \vec{T}\right\rangle d\|T\| d s,
\end{aligned}
$$

and differentiating with respect to $t$ we obtain

$$
\begin{aligned}
& \left.\frac{d}{d t}\right|_{0} \mathbf{M}\left(h_{t \#} T\right)=\int P_{\vec{T}} \cdot D X d\|T\|, \\
& \left.\frac{d}{d t}\right|_{0}\left\langle h_{\#}(\llbracket 0, t \rrbracket \times T), H \Omega\right\rangle=\int H\langle\Omega, X \wedge \vec{T}\rangle d\|T\|,
\end{aligned}
$$

where $X(x)=\partial h / \partial t(0, x)$ is the initial vectorfield of the deformation. We omit the details of these standard calculations (see [Al, Sec. 4], [Fe, 5.1.7], [Si, Section 16]). Since $\langle\Omega, X \wedge \vec{T}\rangle=* \vec{T} \cdot X$ we see that (1.2) follows from (1.1). To prove the converse we define $\left(h_{t}\right)$ by the flow of the vector field $X$. (By approximation it suffices to consider sufficiently smooth vector fields $X$ in (1.1), but the flow of $X$ will satisfy all smoothness requirements for $h$ if $X$ is only $C^{1}$.)

1.4. Remark. Gulliver [Gu2], [Gu3] has derived necessary conditions for the existence of currents with presribed mean curvature (vector). These criteria are based on the variational characterization of the mean curvature. It suffices to describe here a simple special case. Suppose $X$ is a Killing vector field on $N$, i.e. the flow of $X$ is a 1 parameter group of isometries. Then the first variation of $n$ area of any current $T \in \mathcal{R}_{n}(N)$ vanishes, of course, and we have $\int P_{\vec{T}} \cdot D X d\|T\|=0$ if $|D X|$ is $\|T\|$ summable. On the other hand, Killing fields satisfy $0=L_{X} \Omega=d(\Omega\llcorner X)+(d \Omega)\llcorner X=d(\Omega\llcorner X)$ for the Lie derivative $L_{X}$, i.e. $\Omega\llcorner X$ is closed. If $H$ is constant, we can therefore conclude that $\int H(* \vec{T} \cdot X) d\|T\|=H\langle T, \Omega\llcorner X\rangle$ depends only on the homology class of $T$. If we can find $0 \neq T_{0} \in \mathcal{R}_{n}(N)$ homologous to $T$ (i.e. $T=T_{0}+\partial Q$ for some $\left.Q \in \mathcal{R}_{n+1}(N)\right)$ such that $* \vec{T}_{0} \cdot X$ is positive, we therefore must have $\int H(* \vec{T} \cdot X) d\|T\| \neq 0$. This means that equation (1.1) cannot hold. On the other hand, if $X$ vanishes on spt $\partial T=\operatorname{spt} \partial T_{0}$ and $|X|$ is $\|T\|$ summable, then $X$ is (by approximation) an admissible vector field in the definition of mean curvature. When $|X|$ and $|D X|$ are bounded, one concludes that no current $T \in \mathcal{R}_{n}(N)$ of constant mean curvature $H \not \equiv 0$ can exist in $N$ that is homologous to $T_{0}$.

With the obvious choice of the Killing field $X$, this argument can be applied, e.g., to $T_{0}=\llbracket M \times\{z\} \rrbracket$ in a product $N=M \times \mathbb{R}$ or $N=M \times S^{1}$ or to $T_{0}$ representing a great half sphere in $N=S^{n+1}$. No current $T \in \mathcal{R}_{n}(N)$ with constant mean curvature $H \not \equiv 0$ and homologous to $T_{0}$ can exist in these cases. (Theorem 4.6 and 4.7 below shed some light on this non-existence phenomenon from another direction.) Apart from obstructions to the existence of a current 
$T$ with prescribed constant mean curvature $H \not \equiv 0$ that lie in the homology class where one tries to find $T$, there are other obstructions related to the geometry of the boundary. For example, Heinz [He2] proved that the unit circle $S^{1} \times\{0\}$ cannot bound in $\mathbb{R}^{3}$ a surface of constant mean curvature $H$ with $|H|>1$. Gulliver's arguments imply that a similar result holds in the simply connected spaces $N_{\kappa}$ of constant curvature $\kappa^{2}\left(\mathbb{R}^{n+1}, S^{n+1}\right.$ or hyperbolic $n+1$ space if $\kappa^{2}=0,1,-1$ respectively): If $T_{0}$ is represented by a half sphere of radius $r$ in $N_{\kappa}$, then $\partial T_{0}$ cannot be the boundary of a current $T \in \mathcal{R}_{n}\left(N_{\kappa}\right)$ with constant mean curvature $H$ and $|H|$ exceding the mean curvature of this half sphere (which is $n / r$ for $\kappa=0, n \kappa \cot \kappa r$ for $\kappa^{2}>0$ and $n|\kappa| \operatorname{coth}|\kappa| r$ for $\kappa^{2}<0$ ). For the proof one has to use a variational equation for variational vector fields not vanishing on spt $\partial T$ (equation (1.5) in [DS2]). This is satisfied if $T$ minimizes the associated variational functional or if $T$ is sufficiently smooth at the boundary. One then checks that the Killing field argument of [Gu2, Section 4] can be used to prove the claim.

If we impose a constraint spt $T \subset \bar{G}$ on the currents in our variational problem, where $G$ is a smooth domain on $N$ and $N \backslash \bar{G}$ is interpreted as an obstacle that our currents $T \in \mathcal{R}_{n}(N)$ may not penetrate, then we will only know that the left-hand side of (1.2) is nonnegative for all deformations $h$ respecting the constraint, i.e. $h_{t}(\bar{G}) \subset \bar{G}$ for all $t$. This is then, by the proof of Lemma 1.3, equivalent to a variational inequality

$$
\int P_{\vec{T}} \cdot D X d\|T\|+\int H(* \vec{T} \cdot X) d\|T\| \geq 0,
$$

valid for all $C^{1}$ vector fields $X$ on $N$ with compact support in $N \backslash$ spt $\partial T$ such that $X$ is directed weakly inward to $G$ along $\partial G$. In terms of the inner normal $\nu$ of $G$ on $\partial G$, i.e. $\nu=-* \overrightarrow{\partial \llbracket G \rrbracket}$, this latter condition means $X \cdot \nu \geq 0$ on $\partial G$. More precisely, we have the following result:

1.5. Lemma. Suppose $G$ is a $C^{2}$ domain on $N$ with interior unit normal $\nu, H: N \rightarrow \mathbb{R}$ is continuous and $H_{G}(x)$ denotes the mean curvature of the parallel surface to $\partial G$ through $x$ with respect to the inward unit normal at each point $x \in \bar{G}$ where this is defined. Then we have, for $T \in \mathcal{R}_{n}(N)$ with $\operatorname{spt} T \subset \bar{G}$ :

(i) The variational condition

$$
\left.\frac{d}{d t}\right|_{0}\left[\mathbf{M}\left(h_{t \#} T\right)+\left\langle h_{\#}(\llbracket 0, t \rrbracket \times T), H \Omega\right\rangle\right] \geq 0
$$

for all deformations $h$ as in Lemma 1.3 that additionally satisfy $h_{t}(\bar{G}) \subset$ $\bar{G}$, is equivalent to an equation

$$
\int P_{\vec{T}} \cdot D X d\|T\|+\int H(* \vec{T} \cdot X) d\|T\|=\int_{\partial G} \nu \cdot X d \lambda,
$$

for all $C^{1}$ vector fields $X$ with compact support in $N \backslash$ spt $\partial T$, where $\lambda$ is a (nonnegative) Radon measure on $\partial G \backslash$ spt $\partial T$. 
(ii) If the variational condition in (i) holds and $|H| \leq H_{G}$ along $\partial G$, then $\lambda=0$, i.e. $T$ has mean curvature $H$.

(iii) If we have strict inequality $|H|<H_{G}$ along $\partial G$ in (ii); then spt $T \backslash$ spt $\partial T$ is contained in (the interior) $G$.

Here (i) and (ii) were proved in [Du] for the Euclidean situation. The extension to the Riemannian case is straightforward and will be sketched only. The strong inclusion principle (iii), which does only use the variational inequality (1.3) resp. (1.4) and no minimizing property of $T$, was proved for 2 dimensional parametric surfaces in [Hi3], [Hi4]. (For proofs using a minimizing property see [GS2], [DF1], [DS3].)

Proof. (i) We extend $\nu$ to a $C^{1}$ unit vector field on a neighborhood $U$ of $\partial G$ in $N$ such that $C^{2}$ parallel surfaces to $\partial G$ are defined at each $x \in U$ and $\nu$ is perpendicular to these surfaces (with respect to the Riemannian metric of $N)$. Inserting $X=\varphi \nu$ into (1.3) where $\varphi \in C^{1}(N)$ has compact support in $U \backslash$ spt $\partial T$, we obtain the Radon measure $\lambda$ such that the left-hand side of (1.3) equals $\int \varphi d \lambda$. Since (1.3) implies (1.4) in the case $X \cdot \nu=0$ on $\partial G$, the assertion (1.4) then follows from (1.3) in the general case by decomposing $X=(X \cdot \nu) \nu+X-(X \cdot \nu) \nu$ near $\partial G$ into its normal and its tangential part.

(ii) We choose $\varphi \geq 0$ and $X=\varphi(\vartheta \circ \varrho) \nu$ where $\varrho$ is the oriented Riemannian distance function to $\partial G$ and $\vartheta \in C^{1}(\mathbb{R})$ is nonincreasing with $\vartheta \equiv 1$ on $]-\infty, 0$ ] and $\vartheta \equiv 0$ on $[\varepsilon, \infty[$ for $\varepsilon$ so small that $x \in U$ holds for $x \in \operatorname{spt} \varphi$ with $0 \leq$ $\varrho(x) \leq \varepsilon$. Since the Riemannian gradient of $\varrho$ is $\nabla \varrho=\nu$, we have

$$
D X=\varphi(\vartheta \circ \varrho) D \nu+\varphi\left(\vartheta^{\prime} \circ \varrho\right)(\nu \cdot) \otimes \nu+(\vartheta \circ \varrho)(d \varphi) \otimes \nu
$$

and

$$
P_{\vec{T}} \cdot D X=\varphi(\vartheta \circ \varrho) P_{\vec{T}} \cdot D \nu+\varphi\left(\vartheta^{\prime} \circ \varrho\right)\left|P_{\vec{T}} \nu\right|^{2}+(\vartheta \circ \varrho)(\nabla \varphi) \cdot P_{\vec{T}} \nu .
$$

Inserting this into (1.4), making use of $\vartheta^{\prime} \leq 0$, and letting $\vartheta$ approach the characteristic function of $]-\infty, 0]$, we end up with

$$
\int_{\partial G}\left[\varphi P_{\vec{T}} \cdot D \nu+\nabla \varphi \cdot P_{\vec{T}} \nu+\varphi H(* \vec{T} \cdot \nu)\right] d\|T\| \geq \int_{\partial G} \varphi d \lambda .
$$

We now observe that $\operatorname{Tan}(\|T\|, x)=\operatorname{Tan}_{x} \partial G$ for $\|T\|$ almost all $x \in \partial G$, because spt $T \subset \bar{G}$. At such points $x$ we have $P_{\vec{T}} \nu=0$ and $P_{\vec{T}} \cdot D \nu=-H_{G}$, hence the estimate

$$
\lambda \leq\|T\|\left\llcorner\mathbf{1}_{\partial G}\left[H(* \vec{T} \cdot \nu)-H_{G}\right]\right.
$$

follows and proves (ii) in view of $|* \vec{T} \cdot \nu| \leq 1$.

(iii) We first assume that spt $T$ is compact in $\bar{G}$ and spt $\partial T$ contained in $G$. Then the vector field $X=(\varepsilon-\varrho)_{+} \nu$ is admissible in (1.3) and (1.4) for $\varepsilon>0$ sufficiently small (as can be seen by approximation), and the same as in (1.5), (1.6), we obtain

$$
\int_{[\varrho<\varepsilon]}\left[(\varepsilon-\varrho) P_{\vec{T}} \cdot D \nu-\left|P_{\vec{T}} \nu\right|^{2}+(\varepsilon-\varrho) H(* \vec{T} \cdot \nu)\right] d\|T\| \geq 0 .
$$


Consider $x \in U \cap \operatorname{spt} T$ such that $\vec{T}(x)$ is defined and choose an orthonormal basis $\tau_{0}=\nu(x), \tau_{1}, \ldots, \tau_{n}$ in $\operatorname{Tan}_{x} N$. With $\xi:=* \vec{T}(x)$ we then have

$$
\tau_{i}=P_{\vec{T}}(x) \tau_{i}+\left(\xi \cdot \tau_{i}\right) \xi, \quad \tau_{j} \cdot P_{\vec{T}}(x) \tau_{i}=\delta_{i j}-\left(\xi \cdot \tau_{i}\right)\left(\xi \cdot \tau_{j}\right)
$$

Since $\nu$ is a unit vector field and parallel in its own direction (tangent to the shortest geodesics connecting to $\partial G$, in fact), we have $\tau_{0} \cdot D_{\tau_{i}} \nu(x)=0$ and $D_{\tau_{0}} \nu(x)=0 ;$ hence

$$
\begin{aligned}
P_{\vec{T}}(x) \cdot D \nu(x) & =\sum_{i, j=1}^{n}\left(\tau_{j} \cdot P_{\vec{T}}(x) \tau_{i}\right)\left(\tau_{j} \cdot D_{\tau_{i}} \nu(x)\right) \\
& =\sum_{i=1}^{n} \tau_{i} \cdot D_{\tau_{i}} \nu(x)-\sum_{i, j=1}^{n}\left(\xi \cdot \tau_{i}\right)\left(\xi \cdot \tau_{j}\right)\left(\tau_{j} \cdot D_{\tau_{i}} \nu(x)\right) .
\end{aligned}
$$

Here, the first summand is just the negative mean curvature $-H_{G}(x)$ of the parallel surface to $\partial G$ through $x$, and the second term is estimated by $n\|D \nu(x)\| \sum_{i=1}^{n}\left(\xi \cdot \tau_{i}\right)^{2}$. On account of

$$
\sum_{i=1}^{n}\left(\xi \cdot \tau_{i}\right)^{2}=1-\left(\xi \cdot \tau_{0}\right)^{2}=\tau_{0} \cdot P_{\vec{T}}(x) \tau_{0}=\left|P_{\vec{T}}(x) \nu(x)\right|^{2},
$$

we therefore obtain

$$
0 \leq-\int_{[\varrho<\varepsilon]}\left[(\varepsilon-\varrho)\left(H_{G}-|H|\right)+\left|P_{\vec{T}} \nu\right|^{2}(1-(\varepsilon-\varrho) n \sup \|D \nu\|)\right] d\|T\|,
$$

where the supremum is taken on the compact set $[\varrho \leq \varepsilon] \cap \operatorname{spt} T$. With $\varepsilon \downarrow 0$ we infer immediatly spt $T \subset G$ if $|H|<H_{G}$ holds on $\partial G$. (If only $|H| \leq H_{G}$ on a neighborhood of $\partial G$ in $\bar{G}$, we can still conclude that $T$ near $\partial G$ must be the sum of suitably oriented parallel surfaces of $\partial G$.)

To remove the additional hypotheses on $T$, we assume that there exists $x_{0} \in$ $\partial G \cap \operatorname{spt} T \backslash$ spt $\partial G$, and choose a closed Riemannian ball $B_{r}\left(x_{0}\right)$ in $N \backslash$ spt $\partial G$ and a $C^{2}$ domain $\tilde{G} \supset G$ such that $x_{0} \in \partial \tilde{G}$ and $\bar{G} \cap B_{r}\left(x_{0}\right) \backslash\left\{x_{0}\right\} \subset \tilde{G}$, but still $|H|<H_{\tilde{G}}$ on $\partial \tilde{G}$. This may be achieved by defining $\tilde{G}=\Phi_{t}(G)$ for $0<t \ll 1$, where $\Phi_{t}$ is the flow of a smooth compactly supported vector field on $N$ that vanishes at $x_{0}$ and is directed strictly to the exterior of $G$ on $B_{r}\left(x_{0}\right) \cap \partial G \backslash\left\{x_{0}\right\}$. Being guaranteed by (ii) that $T$ has mean curvature $H$, hence (1.3) holds for all $C^{1}$ vector fields $X$ with compact support in $N \backslash \operatorname{spt} \partial T$, we see that $\tilde{T}:=T\left\llcorner B_{r}\left(x_{0}\right)\right.$ satisfies the hypotheses of (iii) with respect to $\tilde{G}$ and that spt $\tilde{T}$ is compact with spt $\partial \tilde{T} \subset \bar{G} \cap B_{r}\left(x_{0}\right) \backslash\left\{x_{0}\right\} \subset \tilde{G}$. We therefore conclude that spt $\tilde{T}$ is contained in $\tilde{G}$ and, in particular, $x_{0} \notin \operatorname{spt} \tilde{T}$. This contradicts the assumption $x_{0} \in \operatorname{spt} T$. 
2. Isoperimetric inequalities. Fundamental for our results on the existence of codimension one currents with prescribed mean curvature in the manifold $N$ (from Section 1) are isoperimetric inequalities of the type

$$
\mathbf{M}(Q) \leq c \mathbf{M}(\partial Q)
$$

or

$$
\mathbf{M}(Q) \leq \gamma \mathbf{M}(\partial Q)^{(n+1) / n}
$$

for $Q \in \mathcal{R}_{n+1}(N)$, possibly required to satisfy certain constraints like mass bounds $\mathbf{M}(Q) \leq r, \mathbf{M}(\partial Q) \leq s$ or an obstacle condition spt $Q \subset \bar{G}$, where $G$ is a smooth domain in $N$. Such inequalities have been extensively studied because of their genuine geometric interest and their relation to the first eigenvalue of the Laplacean of $N$, e.g. Schmidt [Sch], Cheeger [Ch], Gromov [Gr], Hoffmann and Spruck [HSp], Yau [Ya], Croke [Cr1], [Cr2], Kleiner [Kl] (see also [Os], [BZ], [Ga], [GHL] for expositions). In this section we review and supplement some of these results in a form convenient for our setting and later applications.

To establish (2.1) or (2.2) for integer multiplicity currents $Q \in \mathcal{R}_{n+1}(N)$ (with the constraints above), it is sufficient to consider the case of sets with finite perimeter satisfying the same constraints, i.e. $Q=\llbracket A \rrbracket$, where $A \subset \bar{G}$ is $\mathcal{H}^{n+1}$ measurable with finite measure $\mathcal{H}^{n+1}(A)=\mathbf{M}(\llbracket A \rrbracket) \leq r$ and finite total boundary variation (perimeter) $\mathbf{M}(\partial \llbracket A \rrbracket) \leq s$. This assertion is a consequence of the decomposition theorem (cf. [Fe, 4.5.17]), which allows to write $Q=\sum_{i \neq 0}(\operatorname{sign} i) \llbracket A_{i} \rrbracket$ with $\mathbf{M}(Q)=\sum_{i \neq 0} \mathcal{H}^{n+1}\left(A_{i}\right)$ and $\mathbf{M}(\partial Q)=$ $\sum_{i \neq 0} \mathbf{M}\left(\partial \llbracket A_{i} \rrbracket\right)$, where $A_{i}=\left\{x \in N:(\operatorname{sign} i) \zeta_{Q}(x)>|i|\right\}$ for $0 \neq i \in \mathbb{Z}$.

Using standard localisation and smoothing procedures, one can further approximate $A$ by smooth open sets $\Omega$ with compact closure in $\bar{G}$ such that $\Omega \rightarrow A$ in $\mathcal{H}^{n+1}$ measure and $\mathcal{H}^{n}(\partial \Omega) \rightarrow \mathbf{M}(\partial \llbracket A \rrbracket)$. To prove (2.1) or (2.2) for $Q \in \mathcal{R}_{n+1}(N)$ with $\mathbf{M}(Q)<r, \mathbf{M}(\partial Q)<s$ and $\operatorname{spt} Q \subset \bar{G}$, it is therefore sufficient to verify

$$
\begin{gathered}
\mathcal{H}^{n+1}(\Omega) \leq c \mathcal{H}^{n}(\partial \Omega) \\
\mathcal{H}^{n+1}(\Omega) \leq \gamma \mathcal{H}^{n}(\partial \Omega)^{(n+1) / n}
\end{gathered}
$$

respectively for smooth relatively compact domains $\Omega \subset G$ with $\mathcal{H}^{n+1}(\Omega)<r$ and $\mathcal{H}^{n}(\partial \Omega)<s$.

It is well known that isoperimetric inequalities of the type considered above are related to Poincaré and Sobolev inequalities on the manifold N, see e.g. [Au1], [Au2], [MS], [Ya], [HSp]. From the coarea formula

$$
\int_{N}|\nabla f| d \mathcal{H}^{n+1}=\int_{0}^{\infty} \mathbf{M}(\partial \llbracket f>t \rrbracket) d t
$$

for $W^{1,1}$ functions $f \geq 0$ on $N$, one immediately sees that the validity of (2.1) for all $Q \in \mathcal{R}_{n+1}(N)$ with $\operatorname{spt} Q \in \bar{G}$ (or of (2.3) for all smooth domains $\Omega$ with compact closure in $\bar{G}$ ) is equivalent to the Poincaré inequality

$$
\int_{G}|f| d \mathcal{H}^{n+1} \leq c \int_{G}|\nabla f| d \mathcal{H}^{n+1} \quad \text { for } f \in W_{0}^{1,1}(G) .
$$


Here we have abbreviated $[f>t]:=\{x \in N: f(x)>t\}$ and $\llbracket f>t \rrbracket$ for the corresponding $n+1$ current. Using the Fleming and Rishel coarea formula for $f \in B V(N)([\mathrm{Fe}, 4.5 .9(13)],[\mathrm{EG}, 5.5],[\mathrm{Zi}, 5.4 .4])$

$$
\int_{-\infty}^{\infty} \mathbf{M}(\partial \llbracket f>t \rrbracket) d t=\|\partial f\|(N),
$$

one also obtains equivalence to

$$
\int_{G}|f| d \mathcal{H}^{n+1} \leq c\|\partial f\|(\bar{G}) \text { for } f \in B V(N) \text { with spt } f \subset \bar{G} .
$$

To describe the inequalities for $Q \in \mathcal{R}_{n+1}(N)$ or $f \in B V(N)$ that follow from other types of isoperimetric inequalities for sets of finite perimeter, we introduce the isoperimetric norm

$$
|f|:=\sup \int_{N}|f h| d \mathcal{H}^{n+1},
$$

the supremum being taken with respect to all $L_{\text {loc }}^{1}$ functions $h$ on $N$ such that

$$
\int_{A}|h| d \mathcal{H}^{n+1} \leq \mathbf{M}(\partial \llbracket A \rrbracket)
$$

holds for all sets $A \subset N$ of finite perimeter (possibly satisfying constraints $A \subset \bar{G}, \mathbf{M}(\llbracket A \rrbracket) \leq r, \mathbf{M}(\partial \llbracket A \rrbracket) \leq s$ where $G$ is a smooth domain on $N$ and $0<r, s \leq \infty$ are given parameters). One verifies that this defines a norm on the set of $f \in L_{\text {loc }}^{1}(N)$ with $|f|<\infty$ (and spt $f \subset \bar{G}$ ), provided there exists $\delta>0$ such that $\mathcal{H}^{n+1}(A) \leq \delta^{-1} \mathbf{M}(\partial \llbracket A \rrbracket)$ holds for all $A$ with $\mathbf{M}(\partial \llbracket A \rrbracket) \leq \delta$ (and satisfying the constraints). Obviously we have $\left|\mathbf{1}_{A}\right| \leq \mathbf{M}(\partial \llbracket A \rrbracket)$ for sets $A$ with finite perimeter (subject to the constraints), and the isoperimetric norm is the largest norm which has this property and satisfies certain natural conditions.

Using (2.8), $\int_{A} f|h|=\int_{0}^{\infty}\left(\int_{[f>t]}|h|\right) d t$ for $f \geq 0$, and (2.6), we immediately obtain

$$
|f| \leq\|\partial f\|(N)=\mathbf{M}(\partial f)
$$

whenever $0 \leq f \in B V(N)$ (and $[f>t]$ satisfies the constraints for $t>0$ ) or $f$ can be decomposed $f=f_{+}-f_{-}$into functions $f_{+}, f_{-}$with these properties. Inequality (2.9) is a general Sobolev type embedding theorem (cf. [Ma, §2.3]). For example, observing that an isoperimetric inequality

$$
\mathcal{H}^{n+1}(A) \leq c \mathbf{M}(\partial \llbracket A \rrbracket)
$$

implies (2.8) for $h$ with sup $|h| \leq c^{-1}$, we obtain (2.7) again:

$$
\int|f| d \mathcal{H}^{n+1} \leq c|f| \leq c \mathbf{M}(\partial f) .
$$

If, on the other hand,

$$
\mathcal{H}^{n+1}(A) \leq \gamma \mathbf{M}(\partial \llbracket A \rrbracket)^{(n+1) / n}
$$


holds, then (2.8) is valid whenever $\gamma^{n} \int|h|^{n+1} \leq 1$, and we conclude

$$
\int|f|^{(n+1) / n} d \mathcal{H}^{n+1} \leq \gamma|f|^{(n+1) / n} \leq \gamma \mathbf{M}(\partial f)^{(n+1) / n}
$$

for $f \in B V(N)$ as above. This contains the familiar Sobolev inequality

$$
\int_{G}|f|^{(n+1) / n} d \mathcal{H}^{n+1} \leq \gamma\left(\int_{G}|\nabla f| d \mathcal{H}^{n+1}\right)^{(n+1) / n} \quad \text { for } f \in W_{0}^{1,1}(G) .
$$

More generally, if for a Borel function $a:[0, \infty[\rightarrow[0, \infty[$ we define the associated Orlicz type norm

$$
\|f\|_{a}=\sup \left\{\int|f h| d \mathcal{H}^{n+1}: h \in L_{\mathrm{loc}}^{1}(N), \int a \circ|h| d \mathcal{H}^{n+1} \leq 1\right\},
$$

then we have, for $f \in B V(N)$ (with $[f>t]$ and $[f<-t]$ satisfying the constraints for $t>0$ ),

$$
\|f\|_{a} \leq \mathbf{|} f \mathbf{M} \mathbf{M}(\partial f)
$$

whenever $\int a \circ|h| \leq 1$ implies the inequality (2.8) for all sets $A$ of finite perimeter (satisfying the constraints). If $a$ is convex and strictly increasing on $[0, \infty[$, then Jensen's inequality gives

$$
a\left(\frac{1}{|A|} \int_{A}|h| d \mathcal{H}^{n+1}\right) \leq \frac{1}{|A|} \int_{A} a \circ|h| d \mathcal{H}^{n+1} \leq \frac{1}{|A|}
$$

and

$$
\int_{A}|h| d \mathcal{H}^{n+1} \leq|A| a^{-1}\left(\frac{1}{|A|}\right) .
$$

Therefore (2.8) is valid, provided $a$ satisfies an inequality $t a^{-1}(1 / t) \leq b(t)$ for $t>0$ (and $t \leq r)$ where $b$ is any function such that

$$
b\left(\mathcal{H}^{n+1}(A)\right) \leq \mathbf{M}(\partial \llbracket A \rrbracket)
$$

holds for all sets $A \subset N$ with finite perimeter (subject to the constraints); e.g. we may take $b(t)$ as the infimum of values $\mathbf{M}(\partial \llbracket A \rrbracket)$ corresponding to all such sets $A$ with $\mathcal{H}^{n+1}(A) \geq t$ (this function $b$ is called the isoperimetric profile of $\bar{G}$ if $r=s=\infty$; cf. [GHL, 4.74]).

For example, if both isoperimetric inequalities (2.10), (2.12) hold, then we can take

$$
b(t)=\max \left\{\frac{t}{c},\left(\frac{t}{\gamma}\right)^{n /(n+1)}\right\},
$$

and for $a$ we choose take any strictly increasing convex function on $[0, \infty[$ which satisfies

$$
a(t) \geq 0 \text { for } 0 \leq t \leq c^{-1}, \quad a(t) \geq \gamma^{n} t^{n+1} \text { for } t \geq c^{-1} .
$$


(In fact, by an approximation argument $a$ need not be strictly increasing on $[0,1 / c]$.) A natural choice is, with a parameter $0 \leq u \leq c^{-1}$,

$$
a(t)=\gamma^{n} \frac{(t-u)_{+}^{n+1}}{(1-c u)^{n+1}} .
$$

We then find that (2.8) holds for $h$ satisfying

$$
c u+\gamma^{n /(n+1)}\left(\int_{[|h|>u]}(|h|-u)^{n+1} d \mathcal{H}^{n+1}\right)^{1 /(n+1)} \leq 1 \text { for some } u \in\left[0, c^{-1}\right],
$$

but for $\backslash f \backslash$ this gives only the information

$$
\max \left\{\frac{1}{c}\|f\|_{L^{1}}, \gamma^{-n /(n+1)}\|f\|_{L^{(n+1) / n}}\right\} \leq|f|
$$

which we know already from (2.11), (2.13).

There are other choices of $a$, however, which lead to additional restrictions on $|f|$, e.g. $a(t)=0$ for $0 \leq t \leq(n /(n+1))(1 / c), a(t)=\gamma^{n} t^{n+1}$ for $t \geq c^{-1}$, and $a(t)$ affine on $[(n /(n+1))(1 / c),(1 / c)]$. It is elementary to construct $f$ for which the left-hand side in $(2.15)$ is $\leq 1$, but $\|f\|_{a}>1$ and hence also $|f|>1$. The function $a$ here is the smallest convex function satisfying (2.15), but there exist smaller nonconvex functions, e.g.

$$
a(t)= \begin{cases}0 & \text { for } 0 \leq t \leq \frac{n+u}{n+1} \frac{1}{c}, \\ \gamma^{n}\left(\frac{t}{1-u}\right)^{n+1} & \text { otherwise, }\end{cases}
$$

with a parameter $u \in] 0,1\left[\right.$, for which one can still verify that $\int a \circ|h| \leq 1$ implies (2.8) and hence $\|f\|_{a} \leq \mathbf{I} f \mathbf{I}$ is valid. We finally note that for convex, increasing $a$, one can use the conjugate function $\tilde{a}$ and the inequality

$$
\frac{1}{2}\|f\|_{a} \leq \inf \left\{s>0: \int \tilde{a}\left(\frac{1}{s}|f(x)|\right) d \mathcal{H}^{n+1} x \leq 1\right\} \leq\|f\|_{a}
$$

(see $[\mathrm{Ad}, 8.3,8.17])$ to get more explicit inequalities for $f$. For example, we have

$$
\tilde{a}(s)=\max \left\{\frac{n}{c} \frac{s}{n+1}, \frac{n}{\gamma}\left(\frac{s}{n+1}\right)^{(n+1) / n}\right\}
$$

for the convex minorant $a(t)$ above, hence the isoperimetric inequalities (2.10), (2.12) imply the following Sobolev-type inequality

$$
\begin{aligned}
& \int \max \left\{\frac{n}{c s}|f|, \frac{n}{\gamma}\left(\frac{1}{s}|f|\right)^{(n+1) / n}\right\} d \mathcal{H}^{n+1} \leq 1 \\
& \text { for some number } s>0 \text { with } \frac{s}{n+1} \leq\|f\|_{a} \leq \mathbf{|} f \mathbf{|} \leq \mathbf{M}(\partial f) \text {. }
\end{aligned}
$$

The following definition is convenient for our later applications: 
2.1. Definition. For a smooth domain $G \subset N$ and a number $s \in[0, \infty]$ we define the isoperimetric constant $c_{G}(s)$ as the infimum of all real numbers $c \geq 0$ such that $\mathbf{M}(Q) \leq c \mathbf{M}(\partial Q)$ holds for all $Q \in \mathcal{R}_{n+1}(N)$ with spt $Q \subset \bar{G}$ and $\mathbf{M}(\partial Q) \leq s$. The function $c_{G}:[0, \infty] \rightarrow[0, \infty]$ is called the isoperimetric function of $G$.

Clearly, $c_{G}$ is nondecreasing and

$$
\mathbf{M}(Q) \leq c_{G}(\mathbf{M}(\partial Q)) \mathbf{M}(\partial Q)
$$

holds for all $Q \in \mathcal{R}_{n+1}(N)$ with spt $Q \subset \bar{G}$, provided we interprete the righthand side of (2.18) as $\infty$ in the case $c_{G}(\mathbf{M}(\partial Q))=\infty$. In general, $c_{G}$ is neither continuous from the left nor from the right. It is possible that $c_{G}(s)=\infty$ for $s$ finite. In fact, if $N$ has finite volume, then $c_{N}(s)=\infty$ holds for all $s \geq 0$ (consider $Q=\llbracket N \rrbracket)$. Of course, such an isoperimetric function is useless, and we will have to modify the definition when treating compact manifolds $N$ in Section 4. But $c_{N}(s)=\infty$ for finite $s$ may also occur when $N$ has infinite volume.

To produce an example, we choose a sequence of spheres in $\mathbb{R}^{n+2}$ with radii $\geq R>0$ increasing to infinity such that the spheres have distance $\geq 1$ to some hyperplane and disjoint projections onto this hyperplane. Connecting the spheres to the hyperplane by cylindrical tubes of radius $r \in] 0, R]$ (and suitably smoothing out the edges), we obtain a submanifold $N$ of $\mathbb{R}^{n+2}$, diffeomorphic to $\mathbb{R}^{n+1}$, such that $c_{N}(s) \leq c<\infty$ for $s<\omega(n) r^{n}$ and $c_{N}(s)=\infty$ for $s \geq$ $\omega(n) r^{n}$, where $\omega(n)$ is the volume of the unit sphere in $\mathbb{R}^{n+1}$. Note that $N$ may be constructed with bounded second fundamental form and a global tubular neighboorhood in $\mathbb{R}^{n+2}$.

If we modify the previous example by letting the radii of the connecting tubes approach zero as we go through the sequence, then we have $c_{N}(s)=\infty$ for all $s>0$, while $c_{N}(0)=0$. In such a case we cannot prove a general existence result for currents of prescribed mean curvature $H \not \equiv 0$ in $N$. However, this kind of pathological behaviour of the isoperimetric function is excluded if we require some uniformity condition for $N$. A convenient such condition is that $N$ be homogeneously regular in the sense of Morrey, i.e. there exists $\Lambda \in[1, \infty$ [ such that each point in $N$ has a neighborhood that can be mapped to the unit ball in $\mathbb{R}^{n+1}$ by a biLipschitz mapping $\Phi$ with biLipschitz constant $\max \left\{\operatorname{Lip} \Phi, \operatorname{Lip} \Phi^{-1}\right\} \leq \Lambda$. We then have the following result:

2.2. Proposition. Suppose $N$ is homogeneously regular. Then we can find $\delta>0$ and $0<\gamma<\infty$ such that for each $T \in \mathcal{R}_{n}(N)$ with $\partial T=0$ and $\mathbf{M}(T) \leq \delta$ there exists a unique $Q \in \mathcal{R}_{n+1}(N)$ with $\partial Q=T$ and

$$
\mathbf{M}(Q) \leq \gamma \mathbf{M}(T)^{(n+1) / n}
$$

Proof. From the relative isoperimetric inequality [Fe, 4.4.2(2) or 4.5.2(1)] we deduce the following fact: If $U \subset N$ can be mapped onto an open ball in 
$\mathbb{R}^{n+1}$ by a homeomorphism with biLipschitz constant $\leq \Lambda$, then there exists $Q_{U} \in \mathcal{R}_{n+1}(N)$ with spt $Q_{U} \subset \bar{U}$ such that $\left(\partial Q_{U}\right)\llcorner U=T\llcorner U$ and

$$
\mathbf{M}\left(Q\llcorner U) \leq \gamma \mathbf{M}\left(T\llcorner U)^{(n+1) / n},\right.\right.
$$

where $\gamma$ depends only on $\Lambda$ and $n$. Moreover, if $V \supset U$ has the same property as $U$ and $2 \gamma \mathbf{M}(T)<\mathcal{H}^{n+1}(U)$, then we must have $Q_{U}=Q_{V}\llcorner U$ by the constancy theorem.

We now choose a maximal set of points $x_{i} \in N$ with pairwise distance at least $\left(3 \Lambda^{3}\right)^{-1}$ and neighborhoods $V_{i}$ and $U_{i}$ of $x_{i}$, which can be mapped to the unit ball and the concentric ball of radius $\left(3 \Lambda^{2}\right)^{-1}$ in $\mathbb{R}^{n+1}$ respectively by a homeomorphism with biLipschitz constant $\leq \Lambda$ sending $x_{i}$ to the origin. Then the $U_{i}$ cover $N$ and we have $U_{j} \subset V_{i}$ whenever $U_{i} \cap U_{j} \neq \varnothing$. From the observation above we conclude $Q_{U_{i}}\left\llcorner\left(U_{i} \cap U_{j}\right)=Q_{U_{j}}\left\llcorner\left(U_{i} \cap U_{j}\right)\right.\right.$, provided $\mathbf{M}(T) \leq \delta$ and $\delta$ is chosen sufficiently small, depending on $\Lambda$ and $n$. We also note that the number of $j$ such that $U_{j} \cap U_{i} \neq \varnothing$ is bounded by an integer $m$ depending on $\Lambda, n$ only and not on $i$, because the $x_{j} \in V_{i}$ correspond to points with pairwise distance at least $\left(3 \Lambda^{4}\right)^{-1}$ in the unit ball of $\mathbb{R}^{n+1}$.

Choosing a partition of unity $\left(\eta_{i}\right)$ on $N$ subordinate to the covering $\left(U_{i}\right)$ we can now define the locally rectifiable integer multiplicity $n+1$ current $Q=$ $\sum_{i} Q_{U_{i}}\left\llcorner\eta_{i}\right.$ with $\partial Q=T$ and verify with (2.19)

$$
\begin{aligned}
\mathbf{M}(Q) & \leq \sum_{i} \mathbf{M}\left(Q_{U_{i}}\right) \leq \gamma \sum_{i} \mathbf{M}\left(T\left\llcorner U_{i}\right)^{(n+1) / n}\right. \\
& \leq \gamma\left(\sum_{i} \mathbf{M}\left(T\left\llcorner U_{i}\right)\right)^{(n+1) / n} \leq \gamma m^{(n+1) / n} \mathbf{M}(T)^{(n+1) / n}\right.
\end{aligned}
$$

Hence $Q \in \mathcal{R}_{n+1}(N)$, and we have proved the proposition (with $\gamma m^{(n+1) / n}$ instead of $\gamma$ ).

2.3. Corollary. Suppose $G \subset N$ is a domain in $N$, and either $\bar{G}$ is compact with $\bar{G} \neq N$ or $\bar{G}$ is non-compact and $N$ homogeneously regular. Then there exist $\delta>0,0<\gamma<\infty$ such that

$$
c_{G}(s) \leq \gamma s^{1 / n} \quad \text { for } 0 \leq s \leq \delta .
$$

In particular, $c_{G}(s)$ is finite for $0 \leq s \leq \delta$ with limit 0 as $s \downarrow 0$.

Proof. Consider $Q \in \mathcal{R}_{n+1}(N)$ with $\operatorname{spt} Q \subset \bar{G}$ and $\mathbf{M}(\partial Q) \leq s \leq \delta$. Let $\tilde{Q} \in \mathcal{R}_{n+1}(N)$ be the current with $\partial \tilde{Q}=\partial Q$ and $\mathbf{M}(\tilde{Q}) \leq \gamma s^{1+1 / n}$ provided by the proposition. (It is clear from the proof above that we do not need the condition of homogeneous regularity for $N$, if $\bar{G}$ is compact; of course, the constants $\gamma, \delta$ then depend on $\bar{G}$.) In the case $\bar{G}=N$ non-compact 
we have $\mathcal{H}^{n+1}(N)=\infty$ (again by the proof above) and conclude $\tilde{Q}=Q$ from the constancy theorem and from $\mathbf{M}(Q-\tilde{Q}) \leq \mathbf{M}(Q)+\mathbf{M}(\tilde{Q})<\infty$. In the case $\bar{G} \neq N$, the constancy theorem implies $\mathbf{M}(\tilde{Q}) \geq \mathcal{H}^{n+1}(N \backslash \bar{G})>0$ whenever $Q \neq \tilde{Q}$, and this is impossible if we choose $\delta$ to satisfy $\gamma \delta^{1+1 / n}<$ $\mathcal{H}^{n+1}(N \backslash \bar{G})$.

2.4. Remarks. (1) Clearly, $\gamma$ cannot be smaller than the optimal isoperimetric constant $\gamma_{n+1}$ in $\mathbb{R}^{n+1}$, i.e.

$$
\gamma \leq \gamma_{n+1}=\alpha(n+1) \omega(n)^{-1-1 / n}=\frac{1}{n+1} \omega(n)^{-1 / n}
$$

(where $\alpha(n+1)$ is the volume of the unit ball in $\mathbb{R}^{n+1}$ and $\omega(n)=(n+1) \alpha(n+1)$ the $n$ measure of the unit sphere). For simply connected manifolds $N$ of nonpositive sectional curvature and dimension $n+1$, it is conjectured that $\gamma=\gamma_{n+1}$ holds and, in fact, the isoperimetric inequality

$$
\mathbf{M}(Q) \leq \gamma \mathbf{M}(\partial Q)^{(n+1) / n} \quad \text { for all } Q \in \mathcal{R}_{n+1}(N)
$$

is valid with $\gamma=\gamma_{n+1}$. In the case $n=1$ this has been proved by A. Weil and by Beckenbach and Rado for $n=2$ by Kleiner [Kl], and for $n=3$ by Croke [Cr2], who also obtained the best known estimates for the optimal constant $\gamma$ in all other dimensions, namely

$$
\gamma \leq \tilde{\gamma}_{n+1}=\frac{1}{\omega(n)}\left[\omega(n-1) \int_{0}^{\pi / 2} \cos ^{(n+1) /(n-1)} t \sin ^{n-1} t d t\right]^{(n-1) / n}
$$

(2) If $N$ is homogeneously regular, then we have isoperimetric inequalities for $Q \in \mathcal{R}_{n+1}(N)$ with $\mathbf{M}(Q) \leq t$ :

$$
\begin{aligned}
& \mathbf{M}(Q) \leq \gamma(N, t) \mathbf{M}(\partial Q)^{(n+1) / n} \\
& \mathbf{M}(Q) \leq c(N, t) \mathbf{M}(\partial Q)
\end{aligned}
$$

with finite constants $\gamma(N, t), c(N, t)$, provided $t<\mathcal{H}^{n+1}(N)$. In particular, we find $c_{G}(\infty)<\infty$ for the isoperimetric constant of each domain $G \subset N$ with $\mathcal{H}^{n+1}(G)<\mathcal{H}^{n+1}(N)$. To verify this we observe that such inequalities are valid by Proposition 2.2, whenever $\mathbf{M}(\partial Q) \leq \delta$ and $Q$ equals the current $\tilde{Q}$ associated with $\tilde{T}=\partial Q$ by this proposition. Since $\tilde{Q}-Q=k \llbracket N \rrbracket$ for some integer $k$, by the constancy theorem, and we have

$$
\gamma \mathbf{M}(\partial Q)^{(n+1) / n} \geq \mathbf{M}(\tilde{Q}) \geq \mathbf{M}(\tilde{Q}-Q)-\mathbf{M}(Q) \geq|k| \mathcal{H}^{n+1}(N)-t
$$

we see that $Q=\tilde{Q}$ must hold, if $\gamma \mathbf{M}(\partial Q)^{(n+1) / n}<\mathcal{H}^{n+1}(N)-t$. Inequalities (2.20), (2.21) now follow for $\mathbf{M}(\partial Q)$ small (depending on $N$ and $t$ ), while they are trivial for $\mathbf{M}(\partial Q)$ large. 
(3) For general estimates of the isoperimetric constants appearing in (2.20), (2.21) in terms of geometric characteristics of $N$, like (upper) bounds for the sectional curvatures, (lower) bounds for the Ricci curvature, volume (if $N$ is compact), diameter, and injectivity radius, we refer to [Ch], [Ya], [HSp], [Gr], $[\mathrm{Cr} 1],[\mathrm{Cr} 2],[\mathrm{Kl}],[\mathrm{CS}]$, and the expositions of some of these results in [BZ], [Ga], [GHL].

For simply connected manifolds of constant sectional curvature one can use the isoperimetric property of balls to determine the isoperimetric functions and optimal isoperimetric constants.

2.5. Example. Suppose $\kappa$ is real or purely imaginary and $N_{\kappa}$ is the standard $n+1$ dimensional space of constant sectional curvature $\kappa^{2}$ (i.e. $\mathbb{R}^{n+1}$ for $\kappa=0$, the sphere $1 /|\kappa| S^{n+1}$ for $\kappa>0$ and the hyperbolic $n+1$ space with its standard metric multiplied by $1 /|\kappa|$ in the case $\left.\kappa^{2}<0\right)$. Denote by $\alpha_{\kappa}(r)$ the volume $\mathcal{H}^{n+1}\left(B_{r}\right)$ of the ball of radius $r>0$ in $N_{\kappa}$ (where $|\kappa| r<\pi$ if $\kappa^{2}>0$ ) and by $\beta_{\kappa}(r)=\mathcal{H}^{n}\left(\partial B_{r}\right)$ the area of its boundary. We have

$$
\alpha_{\kappa}(r)=\omega(n) \int_{0}^{r}\left(\frac{\sin \kappa \varrho}{\kappa}\right)^{n} d \varrho, \quad \beta_{\kappa}(r)=\alpha_{\kappa}^{\prime}(r)=\omega(n)\left(\frac{\sin \kappa r}{\kappa}\right)^{n},
$$

where, of course, $\kappa^{-1} \sin \kappa r=|\kappa|^{-1} \sinh |\kappa| r$ for $\kappa^{2}<0$ and $\kappa^{-1} \sin \kappa r=r$ for $\kappa=0$. With the differential equation

$$
c_{\kappa}^{\prime}=1-n \kappa(\cot \kappa r) c_{\kappa} \quad \text { for } c_{\kappa}=\frac{\alpha_{\kappa}}{\beta_{\kappa}}
$$

and the power series expansion of $c_{\kappa}(r)$ at $r=0$, one verifies (cf. [BZ, 34.2.2]) that $c_{\kappa}$ is strictly increasing, strictly convex for $\kappa^{2}>0$ and strictly concave for $\kappa^{2}<0$, and

$$
\begin{aligned}
& \frac{\sin \kappa r}{(n+1) \kappa}<c_{\kappa}(r)<\frac{\tan \kappa r}{(n+1) \kappa} \text { if } \kappa^{2}>0,0<2|\kappa| r<\pi, \\
& \frac{\tanh |\kappa| r}{(n+1)|\kappa|}<c_{\kappa}(r)<\min \left\{\frac{\sinh |\kappa| r}{(n+1)|\kappa|}, \frac{\tanh |\kappa| r}{n|\kappa|}\right\} \quad \text { if } \kappa^{2}<0 .
\end{aligned}
$$

We also note $\lim _{r \rightarrow \infty} c_{\kappa}(r)=n^{-1}|\kappa|^{-1}$ if $\kappa^{2}<0$. From the isoperimetric property of balls in $N_{\kappa}$ ([Sch], [DeG], [BZ, 10.2]) we have $\mathbf{M}(\partial \llbracket A \rrbracket) \geq \beta_{\kappa}(r)$ whenever $A \subset N_{\kappa}$ is a set of finite perimeter with $\mathcal{H}^{n+1}(A)=\alpha_{\kappa}(r)$. Using this fact and the stated properties of $c_{\kappa}$ one deduces the following isoperimetric inequalities for $Q \in \mathcal{R}_{n+1}\left(N_{\kappa}\right)$, where the constants are optimal in each case:

$$
\begin{aligned}
& \mathbf{M}(Q) \leq c_{\kappa}(r) \mathbf{M}(\partial Q) \quad \text { if } \mathcal{H}^{n+1}(\operatorname{spt} Q) \leq \alpha_{\kappa}(r), \\
& \mathbf{M}(Q) \leq \frac{1}{n|\kappa|} \mathbf{M}(\partial Q) \quad \text { if } \kappa^{2}<0, \\
& \mathbf{M}(Q) \leq \gamma_{n+1} \mathbf{M}(\partial Q)^{(n+1) / n} \quad \text { if } \kappa^{2} \leq 0,
\end{aligned}
$$




$$
\begin{aligned}
\mathbf{M}(Q) \leq \alpha_{\kappa}(r) \beta_{\kappa}(r)^{-(n+1) / n} \mathbf{M}(\partial Q)^{(n+1) / n} & \\
& \text { if } \kappa^{2}>0 \text { and } \mathcal{H}^{n+1}(\operatorname{spt} Q) \leq \alpha_{\kappa}(r) .
\end{aligned}
$$

Furthermore, we can determine the isoperimetric functions for balls $B_{r}$ in $N_{\kappa}$ (with $0<r<\infty$ if $\kappa^{2} \leq 0,0<|\kappa| r<\pi$ if $\kappa^{2}>0$ ) as follows

$$
c_{B_{r}}(s)= \begin{cases}\frac{1}{s} \alpha_{\kappa}\left(\beta_{\kappa}^{-1}(s)\right) & \text { if } s<\beta_{\kappa}(r), \\ c_{\kappa}(r) & \text { if } s \geq \beta_{\kappa}(r),\end{cases}
$$

where $\beta_{\kappa}^{-1}$ is the inverse function of $\beta_{\kappa}$ (restricted to $\left[0,|2 \kappa|^{-1} \pi\right]$ if $\kappa^{2}>0$ ). Note that $c_{B_{r}}$ is continuous if $\kappa^{2} \leq 0$ or if $2|\kappa| r \leq \pi$, but is discontinuous at $s=\beta_{\kappa}(r)$ if $\kappa^{2}>0$. From the representation

$$
\begin{aligned}
\alpha_{\kappa}\left(\beta_{\kappa}^{-1}(s)\right) & =\int_{0}^{s} \frac{n^{-1} \omega(n)^{-1 / n} \sigma^{1 / n}}{\sqrt{1-\kappa^{2} \omega(n)^{-2 / n} \sigma^{2 / n}}} d \sigma \\
& =\omega(n) \int_{0}^{\sqrt[n]{s / \omega(n)}} \frac{\tau^{n}}{\sqrt{1-\kappa^{2} \tau^{2}}} d \tau
\end{aligned}
$$

we see that $\alpha_{\kappa} \circ \beta_{\kappa}^{-1}$ is strictly increasing and strictly convex. The largest function $b_{\kappa}$, such that $b_{\kappa}(t) \leq \mathbf{M}(\partial \llbracket A \rrbracket)$ for all sets $A \subset N_{\kappa}$ of finite perimeter with $\mathcal{H}^{n+1}(A) \geq t$, is given by $b_{\kappa}=\beta_{\kappa} \circ \alpha_{\kappa}^{-1}$ in the case $\kappa^{2} \leq 0$, and it is strictly increasing and strictly concave. In the case $\kappa^{2}>0$ the same statements hold if we impose the constraint $\mathcal{H}^{n+1}(A) \leq \alpha_{\kappa}(r)$ with $0<|\kappa| r \leq \pi / 2$, while $b_{\kappa}=\min \left\{\beta_{\kappa} \circ \alpha_{\kappa}^{-1}, \beta_{\kappa}(r)\right\}$ if $\pi / 2<|\kappa| r<r$ in this constraint.

Defining $\tilde{a}_{\kappa}(t)$ by the relation $t \tilde{a}_{\kappa}(1 / t)=b_{\kappa}(t)$, we have $\tilde{a}_{\kappa}\left(1 / \alpha_{\kappa}(\varrho)\right)=$ $1 / c_{\kappa}(\varrho)$ for $0<\varrho \leq r^{\prime}$, where $r^{\prime}=\min \left\{r,|\kappa|^{-1} \pi-r\right\}$ in the case $\kappa^{2}>0$ and $r^{\prime}=\infty$ else. We differentiate with the help of $(2.22)$ to see $\tilde{a}_{\kappa}^{\prime}\left(1 / \alpha_{\kappa}(\varrho)\right)=$ $\beta_{\kappa}(\varrho) c_{\kappa}^{\prime}(\varrho)>0$ and

$$
\tilde{a}_{\kappa}^{\prime \prime}\left(\frac{1}{\alpha_{\kappa}(\varrho)}\right)=-n\left(\frac{\sin \kappa \varrho}{\kappa}\right)^{-2} \alpha_{\kappa}(\varrho)^{2} c_{\kappa}(\varrho)<0 .
$$

In the case $\kappa^{2} \leq 0$, it follows that $\tilde{a}_{\kappa}$ has an inverse $a_{\kappa}$ which is strictly increasing and strictly convex. We have $\lim _{t \rightarrow \infty} t^{-1} b_{\kappa}(t)=n|\kappa|$ so that $a_{\kappa}$ is defined on $] n|\kappa|, \infty$ [; we then extend $a_{\kappa}$ continuously (with continuous derivative, in fact, since $t^{-2} \tilde{a}_{\kappa}(1 / t) \rightarrow-\infty$ as $\left.t \rightarrow \infty\right)$ to $[0, \infty$ [ by the value 0 on $[0, n|\kappa|]$. Recalling (2.14), we obtain the Sobolev-type inequality $\|f\|_{a_{\kappa}} \leq \mathbf{M}(\partial f)$. Since $a_{\kappa}(t) \leq \gamma_{n+1}^{n} t^{n+1}$ for $t>n|\kappa|$, by (2.23), we see in particular

$$
\int_{[|h|>n|\kappa|]}|h|^{n+1} d \mathcal{H}^{n+1} \leq \gamma_{n+1}^{-n} \Longrightarrow \int_{N_{\kappa}}|f h| d \mathcal{H}^{n+1} \leq \mathbf{M}(\partial f) .
$$

This inequality is stronger than what we have derived earlier from the isoperimetric inequalities (2.10), (2.12) (cf. (2.17) and the preceding discussion). 
In the case $\kappa^{2}>0$ one can proceed similarly, provided the constraint $\mathcal{H}^{n+1}(A) \leq \alpha_{\kappa}(r)$ is imposed with $0<|\kappa| r<\pi$. The function $a_{\kappa}(t)$ is then strictly increasing and convex for $t \geq 1 / c_{\kappa}(r)$, strictly convex for $t \geq 1 / c_{\kappa}\left(r^{\prime}\right)$, and affine for $1 / c_{\kappa}(r) \leq t \leq 1 / c_{\kappa}\left(r^{\prime}\right)$. Extending $a_{\kappa}$ to a nonnegative, increasing convex function on $\left[0, \infty\left[\right.\right.$ we obtain the Sobolev type inequality $\|f\|_{a_{\kappa}} \leq \mathbf{M}(\partial f)$ for $B V$ functions $f$ on $N_{\kappa}$ with $\mathcal{H}^{n+1}(\operatorname{spt} f) \leq \alpha_{\kappa}(r)$. However, if we replace $a_{\kappa}$ by a majorant using (2.23), this time we do not obtain a better inequality than $(2.16)$.

A simple idea to prove an isoperimetric inequality of type (2.1) is the construction of a vectorfield $X$ such that $|X| \leq 1$ and $\operatorname{div} X \geq c^{-1}$ holds on $N$. Then, by the divergence theorem,

$$
\frac{1}{c} \mathcal{H}^{n}(\Omega) \leq \int_{\Omega} \operatorname{div} X d \mathcal{H}^{n+1}=-\int_{\partial \Omega} X \cdot \nu d \mathcal{H}^{n} \leq \mathcal{H}^{n}(\partial \Omega)
$$

will hold for smooth relatively compact domains $\Omega \subset N$ with interior unit normal $\nu$. In fact, it is sufficient to know that $X$ is $\mathcal{H}^{n+1}$ measurable with $|X| \leq 1$ almost everywhere and $\operatorname{div} X \geq c^{-1}$ in the sense of disributions on the open set $G \subset N$ to infer $\mathcal{H}^{n+1}(A) \leq c \mathbf{M}(\partial \llbracket A \rrbracket)$ for all sets $A$ with finite perimeter and $\bar{A} \subset G$, and, hence, $\mathbf{M}(Q) \leq c \mathbf{M}(\partial Q)$ for all $Q \in \mathcal{R}_{n+1}(N)$ with spt $Q \subset G$. (In the case of a sufficiently smooth domain $G$ we will have the same inequality also for $\bar{A} \subset \bar{G}$ or spt $Q \subset \bar{G}$.) This follows from the approximation arguments at the beginning of this section, because in the inequality

$$
\frac{1}{c} \int_{G} \varphi d \mathcal{H}^{n+1} \leq-\int_{G} X \cdot \nabla \varphi d \mathcal{H}^{n+1} \leq \int_{G}|\nabla \varphi| d \mathcal{H}^{n+1}
$$

we can let the smooth function $\varphi \geq 0$ with compact support in $G$ converge to the characteristic function of $A$ such that the first integral tends to $\mathcal{H}^{n+1}(A)$ and the last to $\mathbf{M}(\partial \llbracket A \rrbracket)$.

In much of the work on parametric surfaces with prescribed mean curvature the idea above has been used in implicit form by constructing a vector field $X$ such that $|X| \leq 1$ and $\operatorname{div} X$ equals the prescribed mean curvature function $H$. The field $X$ was defined via an integral formula involving $H$ or through solution of the equation for nonparametric surfaces of prescribed mean curvature

$$
\operatorname{div} \frac{\nabla u}{\sqrt{1+|\nabla u|^{2}}}=H
$$

and then setting $X=\left(1+|\nabla u|^{2}\right)^{-1 / 2} \nabla u$ (see e.g. [Hi2], [GS2], [Gu1]). Our approach here has more flexibility, because we need only satisfy the inequality $\operatorname{div} X \geq H$ (when $H \geq 0$ ). In the context of parametric surfaces in $\mathbb{R}^{3}$ this approach was introduced in [St1], [St2], and for hypersurfaces of prescribed mean curvature in $\mathbb{R}^{n+1}$ it was used in $[\mathrm{Du}]$ in the setting of integer multiplicity rectifiable currents.

A natural choice for $X$ is the radial unit vector field $X\left(\exp _{a} \xi\right)=$ $\left(d_{\xi} \exp _{a}\right) \xi /|\xi|$ on a normal neighborhood $G$ of a point $a \in N$, i.e. $G$ is the 
diffeomorphic image of a star-shaped (with respect to the origin) domain in $\operatorname{Tan}_{a} N$ under the exponential map. Since $X$ is parallel in its own direction, we see that $\operatorname{div} X=\operatorname{trace} D X$ at $x \in G \backslash\{a\}$ is just the mean curvature $h(x)$ of a spherical hypersurface $S$ through $x$ (i.e. the image $S=\exp _{a} \Sigma$ of a neighborhood $\Sigma$ of $\xi=\exp _{a}^{-1}(x)$ in the sphere of radius $|\xi|$ about the origin in $\operatorname{Tan}_{a} N$; the mean curvature $h(x)$ is taken with respect to the "interior" normal $-X(x)$ to $S$ at $x$ ). Using $|X| \leq 1$ and $n+1 \geq 2$, one sees easily that $\operatorname{div} X=h$ on $G$ holds in the sense of distributions.

On the other hand, employing Remark 1.2, (1) with the variation $S_{t}=$ $\exp _{a}\left(e^{t} \Sigma\right)$, we get $\int_{S} h d \mathcal{H}^{n}=d /\left.d t\right|_{t=0} \mathcal{H}^{n}\left(S_{t}\right)$, and letting $S$ shrink to $\{x\}$, we infer

$$
h(x)=\frac{\left.\frac{d}{d t}\right|_{t=0} J\left(e^{t} \xi\right)}{J(\xi)},
$$

where $J(\xi)$ denotes the Jacobian of $\exp _{a}$ at $\xi \in \operatorname{Tan}_{a} N$ (this is also the Jacobian of the restriction of $\exp _{a}$ to the sphere with radius $\left.|\xi|\right)$. In particular, for the constant mean curvature $h_{\kappa}(\varrho)$ of the spheres with radius $\varrho$ in a space $N_{\kappa}$ of constant curvature $\kappa^{2}$ we find the value

$$
h_{\kappa}(\varrho)=\beta_{\kappa}(\varrho) \frac{d}{d \varrho} \beta_{\kappa}(\varrho)=n \kappa \cot \kappa \varrho
$$

(see Example 2.5; we assume $|\kappa| \varrho<\pi$ when $\kappa^{2}>0$; note $n \kappa \cot \kappa \varrho=n / \varrho$ if $\kappa=$ 0 and recall our convention that the mean curvature is the sum of the principal curvatures). For general $N$ with sectional curvatures $\leq \kappa^{2}$ the comparison theorem for Jacobians, ([Gü], [BZ, Section 33], [GHL, 3.101]) asserting $h(x) \geq$ $h_{\kappa}(|\xi|)$ for $x=\exp _{a} \xi$, now implies the following proposition which is well known. The corollary was apparently first stated by Yau [Ya].)

2.6. Proposition. Suppose $N$ has sectional curvatures $\leq \kappa^{2}$ and $G$ is a normal neighborhood of $a \in N$ such that each geodesic arc in $G$ emanating from a has length less than $r$ (with $2|\kappa| r<\pi$ if $\kappa^{2}>0$ ). Then an isoperimetric inequality $\mathbf{M}(Q) \leq c \mathbf{M}(\partial Q)$ holds for all $Q \in \mathcal{R}_{n+1}(N)$ with $\operatorname{spt} Q \subset G$, where the constant is $c=(n \kappa)^{-1} \tan \kappa r$.

2.7. Corollary. If $N$ is simply connected with sectional curvatures $\leq \kappa^{2}<$ 0 , then we have $\mathbf{M}(Q) \leq(n|\kappa|)^{-1} \mathbf{M}(\partial Q)$ for all $Q \in \mathcal{R}_{n+1}(N)$.

2.8. Remarks. (1) Actually we have shown, with the hypotheses above,

$$
\int_{A} n \kappa \cot \kappa \varrho(x) d \mathcal{H}^{n+1} x \leq \int_{A} h(x) d \mathcal{H}^{n+1} x \leq \mathbf{M}(\partial \llbracket A \rrbracket)
$$

for sets $A$ of finite perimeter with $\bar{A} \subset G$, where $\varrho(x)$ denotes the length of the geodesic segment in $G$ connecting $x$ with $a$.

(2) We can improve the constant $c$ in Proposition 2.6 by considering a vector field $\tilde{X}(x)=\eta(\varrho(x)) X(x)$. Using $\nabla \varrho=X$, we find

$$
\operatorname{div} \tilde{X}=\eta^{\prime}(\varrho)+\eta(\varrho) \operatorname{div} X \geq \eta^{\prime}(\varrho)+\eta(\varrho) n \kappa \cot \kappa \varrho
$$


in the sense of distributions on $G$, and choosing $\eta(\varrho)=c_{\kappa}(\varrho)$, we obtain $\operatorname{div} \tilde{X} \geq$ 1 from $(2.22)$. Since $c_{\kappa}$ is increasing, the inequality

$$
\mathbf{M}(Q) \leq c_{\kappa}(r) \mathbf{M}(\partial Q)
$$

follows for $Q \in \mathcal{R}_{n+1}(N)$ with spt $Q \subset G$ (and $|\kappa| r<\pi$ if $\kappa^{2}>0$ ), and the constant $c_{\kappa}(r)$ is smaller then the constant $c=(n \kappa)^{-1} \tan \kappa r$ in the Proposition2.6, e.g. $c_{\kappa}(r)=r /(n+1)$ and $c=r / n$ in the case $\kappa=0$ (cf. Example 2.5). We may rephrase $(2.27)$ by saying that on normal domains with maximal radius $<r$ in manifolds $N$ with sectional curvatures $\leq \kappa^{2}$ the same "linear" isoperimetric inequality holds as on geodesic balls of radius $r$ in the space $N_{\kappa}$ of constant curvature $\kappa^{2}$.

(3) With one-sided curvature restrictions for $N$ as in Proposition 2.6 we cannot derive "nonlinear" isoperimetric inequalities like (2.2). However, if we assume, in addition to the hypotheses of Proposition 2.6, a lower bound $n \lambda^{2}$ for the Ricci curvatures of $N$ (where $\lambda \in \mathbb{R} \cup \sqrt{-1} \mathbb{R}$ with $\lambda^{2} \leq \kappa^{2}$ ), then from (2.23) and the comparison theorem of Rauch and Bishop ([BZ, Section 32, Section 33], [GHL, 3.101]) we see that

$$
\mathbf{M}(Q) \leq\left(\frac{\kappa \sin \lambda r}{\lambda \sin \kappa r}\right)^{n} \gamma_{\kappa}(r) \mathbf{M}(\partial Q)^{(n+1) / n}
$$

holds for all $Q \in \mathcal{R}_{n+1}(N)$ with spt $Q \subset G$, where $\gamma_{\kappa}(r)=\gamma_{n+1}$ and $\gamma_{\kappa}(r)=$ $\alpha_{\kappa}(r) \beta_{\kappa}(r)^{-1-1 / n}$ in the cases $\kappa^{2} \leq 0$ and $\kappa^{2}>0$ respectively.

2.9. Proposition. Suppose $G \subset N, G \neq N$, is a $C^{2}$ domain such that its mean curvature function satisfies $H_{G} \geq c^{-1}>0$ on its domain of definition. Then the isoperimetric inequality $\mathbf{M}(Q) \leq c \mathbf{M}(\partial Q)$ is valid for all $Q \in \mathcal{R}_{n+1}(N)$ with spt $Q \subset \bar{G}$.

Proof. We denote by $\nu$ the interior unit normal field on $\partial G$ and we consider the exponential map $\exp _{\partial G}: t \nu(x) \mapsto \exp _{x} t \nu(x)$ of the normal bundle $\operatorname{Tan}^{\perp} \partial G$ of $\partial G$ in $N$. For $x \in \partial G$ we denote by $\tau(x) \leq \infty$ the supremum of numbers $t>0$ such that $\exp _{\partial G}$ maps the segment $[0, t] \nu(x)$ with maximal rank onto a geodesic arc in $\bar{G}$ which is the unique shortest connection between $\exp _{\partial G} t \nu(x)$ and $\partial G$. It is well known that $\exp _{\partial G} \operatorname{maps} \operatorname{graph}(\tau)=\{\tau(x) \nu(x): x \in \partial G, \tau(x)<\infty\}$ onto a closed set $C \subset G$ (the cut locus of $\partial G$ ), while $\operatorname{kat}(\tau)=\{t \nu(x): x \in \partial G, 0<$ $t<\tau(x)\}$ is mapped diffeomorphically onto $G \backslash C$. We have $\mathcal{H}^{n+1}(C)=0$, because graph $(\tau)$ has $\mathcal{H}^{n+1}$ measure zero in $\operatorname{Tan}^{\perp} \partial G$. We can therefore extend $-\nu$ to a smooth unit vector field $X$ defined $\mathcal{H}^{n+1}$ almost everywhere on $\bar{G}$ by requiring that $X(x)$ at $x \in G \backslash C$ is tangent to the unique shortest geodesic arc connecting $x$ with $\partial G$. Then $\operatorname{div} X=\operatorname{trace} D X$ is just the mean curvature function $H_{G}$ of $G$ introduced in Lemma 1.5, i.e. $H_{G}(x)$ is the mean curvature of the parallel surface to $\partial G$ at $x \in \bar{G} \backslash C$ with respect to its inward normal $-X(x)$.

As we have seen above, it is sufficient to verify that $\operatorname{div} X \geq c^{-1}$ holds on $G$ in the sense of distributions. For this we observe that $\tau$ is lower semicontinous 
on $\partial G$ and hence we can choose an increasing sequence $\tau_{k}$ of smooth positive functions on $\partial G$ which converge pointwise to $\tau$. We denote by $G_{k}$ the increasing sequence of open sets $\exp _{\partial G}\left(\right.$ kat $\left.\tau_{k}\right)$ with union $G \backslash C$. Applying the divergence theorem to $G_{k}$, we get, for smooth functions $\varphi \geq 0$ with compact support in $G$,

$$
\begin{aligned}
\frac{1}{c} \int_{G_{k}} \varphi d \mathcal{H}^{n+1} & \leq \int_{G_{k}} \varphi H_{G} d \mathcal{H}^{n+1}=\int_{G_{k}} \varphi \operatorname{div} X d \mathcal{H}^{n+1} \\
& =-\int_{G_{k}} X \cdot \nabla \varphi d \mathcal{H}^{n+1}-\int_{\partial G_{k}} \varphi X \cdot \nu_{k} d \mathcal{H}^{n},
\end{aligned}
$$

where $\nu_{k}$ is the interior unit normal of $G_{k}$ on $\partial G_{k}$. We have $\partial G \cap \operatorname{spt} \varphi=\varnothing$ and $X \cdot \nu_{k}>0$ on $\partial G_{k} \backslash \partial G=\exp _{\partial G}\left(\right.$ graph $\left.\tau_{k}\right)$, hence passing to the limit $k \rightarrow \infty$ and recalling $\mathcal{H}^{n+1}(C)=0$, we obtain the claim $\operatorname{div} X \geq c^{-1}$ on $G$.

2.10. Remarks. (1) We have actually proved the inequality

$$
\int_{A}|H| d \mathcal{H}^{n+1} \leq \mathbf{M}(\partial \llbracket A \rrbracket)
$$

for all sets $A \subset \bar{G}$ of finite perimeter if $|H| \leq H_{G}$ holds on $G$. Using the vector field $(1-a \operatorname{dist}(\cdot, \partial G)) X$ with a parameter $0 \leq a<\inf _{G} \operatorname{dist}(\cdot, \partial G)^{-1}$ as in [GS2], [Se], we can establish the same inequality if

$$
|H| \leq(1-a \operatorname{dist}(\cdot, \partial G)) H_{G}+a
$$

holds on $G$.

(2) With the comparison theorem of Heintze-Karcher [HeK] (cf. [BZ, 33.3]) one can reduce the verification of the hypothesis $H_{G} \geq c^{-1}$ on a geodesic arc $\gamma(t)=\exp _{\partial G} t \nu(x), 0 \leq t<\tau(x)$, to the corresponding inequality on a geodesic arc $\tilde{\gamma}(t)$ in a space $N_{\kappa}$ of constant curvature which is perpendicular at $\tilde{x}=\tilde{\gamma}(0)$ to a hypersurface $\tilde{S}$ in $N_{\kappa}$, provided $N$ has sectional curvatures $\leq \kappa^{2}$, the principal curvatures of $\partial G$ at $x$ are (one by one) not smaller than those of $\tilde{S}$ at $\tilde{x}$, and the first cut point of $\partial G$ on $\gamma$ does not appear later than the first focal point of $\tilde{S}$ on $\tilde{\gamma}$.

We conclude our discussion of isoperimetric inequalities with a result for the isoperimetric function of a product $N=M \times \mathbb{R}$ of a closed $n$ dimensional Riemannian manifold $M$ with the Euclidean line which will be of interest later.

2.11. Proposition. Suppose $N=M \times \mathbb{R}$ where $M$ is compact (without boundary). Then the isoperimetric function $c_{N}(s)$ is infinite for $s \geq 2 \mathcal{H}^{n}(M)$ and bounded from above by a finite constant for $0 \leq s<2 \mathcal{H}^{n}(M)$.

The proof will actually give an explicit expression for this constant in terms of $n, \mu$ and the isoperimetric constants $\gamma(M, \mu / 2), c(M, 3 \mu / 4)$ from $(2.20),(2.21)$ for $M$, where $\mu=\mathcal{H}^{n}(M)$ is the volume of $M$. 
Proof. First observe that $A_{t}=M \times[0, t]$ for $t>0$ is a set with finite perimeter in $N$ such that $\mathbf{M}(\partial \llbracket A \rrbracket)=2 \mu$ and $\mathcal{H}^{n+1}\left(A_{t}\right)=t \mu$, hence $c_{N}(s)=\infty$ for $s \geq 2 \mu$ follows with $t \rightarrow \infty$. Consider now a smooth domain $\Omega \subset N$ with compact closure and $\mathcal{H}^{n}(\partial \Omega)<2 \mu$. Since, by Sard's theorem, for $\mathcal{H}^{n}$ almost all $x \in M$ the line $\{x\} \times \mathbb{R}$ intersects $\partial \Omega$ transversally and, hence, in an even number of points, we see that the projection of $\bar{\Omega}$ onto $M$ is a set of $\mathcal{H}^{n}$ measure $\tilde{\mu}<\mu$ and so are all the slices $\Omega_{t}=\{x \in M:(x, t) \in \Omega\}$. In the case $\mathcal{H}^{n}\left(\Omega_{t}\right) \leq 3 \mu / 4$ for all $t$ we use the isoperimetric inequality (2.21) on $M$ with $c=c(M, 3 \mu / 4)$ and the coarea formula to deduce

$$
\mathcal{H}^{n+1}(\Omega)=\int_{-\infty}^{\infty} \mathcal{H}^{n}\left(\Omega_{t}\right) d t \leq c \int_{-\infty}^{\infty} \mathcal{H}^{n-1}\left(\partial \Omega_{t}\right) d t \leq c \mathcal{H}^{n}(\partial \Omega) .
$$

We now consider the case where $\mathcal{H}^{n}\left(\Omega_{t_{0}}\right)>3 \mu / 4$ for some $t_{0}$, and we chose $t_{0}$ such that $(1+\varepsilon)^{-1} \mathcal{H}^{n}\left(M \backslash \Omega_{t_{0}}\right)$ for a given $\varepsilon>0$ does not exceed $\inf _{t \in \mathbb{R}} \mathcal{H}^{n}\left(M \backslash \Omega_{t}\right)$. (This is possible, because $\mathcal{H}^{n}\left(M \backslash \Omega_{t}\right) \geq \mu-\tilde{\mu}>0$ for all $t$.) Our aim is to estimate $t_{1}-t_{0}$ when $\mathcal{H}^{n}\left(\Omega_{t_{1}}\right)>3 \mu / 4$ for some $t_{1}>t_{0}$. For this we first observe that $\mathcal{H}^{n}\left(\Omega_{t}\right)>\mu / 2$ holds for all $\left.t \in\right] t_{0}, t_{1}\left[\right.$ with $\mathcal{H}^{n}\left(\partial \Omega_{t}\right)=0$, because for $\mathcal{H}^{n}$ almost all $x \in \Omega_{t_{1}} \backslash \bar{\Omega}_{t}$ the half line $\left.\{x\} \times\right] t, \infty[$ intersects $\partial \Omega$ in at least two points and for $\mathcal{H}^{n}$ almost all $x \in \Omega_{t_{1}}$ in at least one point so that

$$
\mathcal{H}^{n}(\partial \Omega \cap M \times] t_{1}, \infty[) \geq 2 \mathcal{H}^{n}\left(\Omega_{t_{1}}\right)-\mathcal{H}^{n}\left(\Omega_{t}\right) \geq \frac{3}{2} \mu-\mathcal{H}^{n}\left(\Omega_{t}\right)
$$

holds and similarly also with $] t, \infty\left[, t_{1}\right.$ replaced by $]-\infty, t\left[, t_{0}\right.$, hence $2 \mathcal{H}^{n}\left(\Omega_{t}\right) \geq$ $3 \mu-\mathcal{H}^{n}(\partial \Omega)>\mu$. From the choice of $t_{0}$, the coarea formula, and the isoperimetric inequality $(2.20)$ on $M$ with $\gamma=\gamma(M, \mu / 2)$, we now obtain, for $t \in] t_{0}, t_{1}[$,

$$
\begin{aligned}
(2+\varepsilon) \mathcal{H}^{n}\left(M \backslash \Omega_{t}\right) \geq \mathcal{H}^{n}\left(M \backslash \Omega_{t_{0}}\right)+\mathcal{H}^{n}\left(M \backslash \Omega_{t}\right) \\
\quad \geq 2 \mathcal{H}^{n}(M)-\mathcal{H}^{n}\left(\Omega_{t_{0}}\right)-\mathcal{H}^{n}\left(\Omega_{t}\right)>\mathcal{H}^{n}(\partial \Omega)-\mathcal{H}^{n}\left(\Omega_{t_{0}}\right)-\mathcal{H}^{n}\left(\Omega_{t}\right) \\
\quad \geq \int_{t_{0}}^{t} \mathcal{H}^{n-1}\left(\partial \Omega_{s}\right) d s \geq \int_{t_{0}}^{t}\left[\frac{1}{\gamma} \mathcal{H}^{n}\left(M \backslash \Omega_{s}\right)\right]^{(n-1) / n} d s,
\end{aligned}
$$

which is equivalent to the differential inequality

$$
\eta^{\prime}(t) \geq\left(\frac{\eta(t)}{\gamma(2+\varepsilon)}\right)^{(n-1) / n} \quad \text { for } \eta(t)=\int_{t_{0}}^{t}\left[\frac{1}{\gamma} \mathcal{H}^{n}\left(M \backslash \Omega_{s}\right)\right]^{(n-1) / n} d s
$$

Integrating this inequality, using (2.29) to estimate $\eta\left(t_{1}\right) \leq(2+\varepsilon) \mathcal{H}^{n}\left(M \backslash \Omega_{t_{1}}\right) \leq$ $(2+\varepsilon) \mu / 4$, and letting $\varepsilon \downarrow 0$, we arrive at

$$
t_{1}-t_{0} \leq \frac{1}{2} n(4 \gamma)^{1-1 / n} \mu^{1 / n} .
$$

From (2.30) and a similar discussion for $t_{1}<t_{0}$, we conclude that the set of $t \in \mathbb{R}$ with $\mathcal{H}^{n}\left(\Omega_{t}\right)>3 \mu / 4$ is contained in an interval $I$ with length twice the 
constant in (2.30). We can therefore estimate

$$
\begin{aligned}
\mathcal{H}^{n+1}(\Omega) & =\int_{\mathbb{R} \backslash I} \mathcal{H}^{n}\left(\Omega_{t}\right) d t+\int_{I} \mathcal{H}^{n}\left(\Omega_{t}\right) d t \\
& \leq \int_{\mathbb{R} \backslash I} c \mathcal{H}^{n-1}\left(\partial \Omega_{t}\right) d t+n(4 \gamma)^{1-1 / n} \mu^{1 / n} \mathcal{H}^{n}(M) \\
& \leq\left(c+\frac{2}{3} n(4 \gamma)^{1-1 / n} \mu^{1 / n}\right) \mathcal{H}^{n}(\partial \Omega),
\end{aligned}
$$

where we have taken into account $\mathcal{H}^{n}(\partial \Omega) \geq 2 \mathcal{H}^{n}\left(\Omega_{t_{0}}\right) \geq 3 \mu / 2=3 \mathcal{H}^{n}(M) / 2$. This inequality together with inequality (2.28) from the first case proves the proposition.

It is clear that the proposition is valid for more general Riemannian metrics on $N=M \times \mathbb{R}$ than the product metric, e.g. for any metric which is biLipschitz equivalent to the product metric.

3. Existence in non-closed manifolds. The equation (1.1) for currents $T \in \mathcal{R}_{n}(N)$ of prescribed mean curvature $H$ in $N$ has a variational structure. Therefore, to prove existence of such currents with given boundary $\partial T=\partial T_{0}$, where $T_{0}$ is a given reference current, we shall apply the direct method of the calculus of variations. From Lemma 1.3 one sees that the energy functional $\mathbf{E}_{H}(T)$, which we have to minimize, must be of the form $\mathbf{E}_{H}(T)=\mathbf{M}(T)+$ $\mathbf{V}_{H}\left(T, T_{0}\right)$, where $\mathbf{V}_{H}\left(T, T_{0}\right)$ in some sense is the volume enclosed by $T$ and $T_{0}$ with respect to the weight function $H$. More precisely, we should have

$$
\mathbf{V}_{H}\left(T, T_{0}\right)=\langle H Q, \Omega\rangle=\int H \zeta_{Q} d \mathcal{H}^{n+1},
$$

where $Q \in \mathcal{R}_{n+1}(N)$ has boundary $\partial Q=T-T_{0}$ (and integer valued multiplicity function $\zeta_{Q} ; \Omega$ is the volume form of $N$, see Section 1 for notations). In order to make this a reasonable definition of volume, we must know that $Q$ is uniquely determined by $T$ and $T_{0}$. By the constancy theorem, this will be the case if $N$ has infinite volume or if we require that $T, T_{0}, Q$ have supports in the closure of a domain $G \subset N$ with $\bar{G} \neq N$. We refer to this situation as the case of a "non-closed" ambient space, because the case of a closed manifold (i.e. compact without boundary) is excluded. (In the latter case $Q$ will not be unique and the reasoning has to be modified; see Section 4.)

As general assumptions for this chapter, we therefore require that $N$ be oriented, connected and complete of dimension $n+1$, as always, and $G$ is a $C^{2}$ domain on $N$ such that $\bar{G} \neq N$ or $G=N$ is non-compact with $\mathcal{H}^{n+1}(N)=\infty$. In the case where $\bar{G}$ is non-compact we sometimes require that $N$ is homogeneously regular (cf. Proposition 2.2) and, hence, the volume $\mathcal{H}^{n+1}(N)$ is infinite. However, it is clear from the proofs that many of the following results are also true without this uniformity condition. For the prescribed mean curvature function $H: \bar{G} \rightarrow \mathbb{R}$ we always assume continuity and boundedness. 


\subsection{Definitions.}

(i) For $T_{0} \in \mathcal{R}_{n}(N)$ with spt $T_{0} \subset \bar{G}$, the class of admissible currents $\mathcal{C}\left(T_{0}, G\right)$ consists of all $T \in \mathcal{R}_{n}(N)$ that are homologous to $T_{0}$ in $\bar{G}$, i.e. there exists $Q \in \mathcal{R}_{n+1}(N)$ with spt $Q \subset \bar{G}$ and $\partial Q=T-T_{0}$ (in particular $\partial T=\partial T_{0}$ and $\operatorname{spt} T \subset \bar{G})$. For $1<\sigma \leq \infty$, we set $\mathcal{C}\left(T_{0}, G, \sigma\right):=\left\{T \in \mathcal{C}\left(T_{0}, G\right)\right.$ : $\left.\mathbf{M}(T) \leq \sigma \mathbf{M}\left(T_{0}\right)\right\}$.

(ii) For $T \in \mathcal{C}\left(T_{0}, G\right)$ we define the $H$ volume

$$
\mathbf{V}_{H}\left(T, T_{0}\right)=\langle Q, H \Omega\rangle=\int H \zeta_{Q} d \mathcal{H}^{n+1},
$$

where $Q$ is the (unique, by the general assumptions for this section) current in $\mathcal{R}_{n+1}(N)$ with spt $Q \subset \bar{G}$ and $\partial Q=T-T_{0}$. The $H$ energy of $T$ is then $\mathbf{E}_{H}(T)=\mathbf{M}(T)+\mathbf{V}_{H}\left(T, T_{0}\right)$.

(iii) By an isoperimetric condition of type $(c, s)$ for $H$ on $\bar{G}$, we mean an inequality

$$
\left|\int_{A} H d \mathcal{H}^{n+1}\right| \leq c \mathbf{M}(\partial \llbracket A \rrbracket)
$$

for all sets $A \subset \bar{G}$ of finite perimeter with $\mathbf{M}(\partial \llbracket A \rrbracket) \leq s$. (Here we assume $0<c<\infty$ and $0<s \leq \infty$ for the parameters.)

We note that, by the decomposition theorem (cf. (2.8) and (2.9)), an equivalent formulation of the isoperimetric condition is

$$
\left|\int H \zeta_{Q} d \mathcal{H}^{n+1}\right| \leq c \mathbf{M}(\partial Q)
$$

$$
\text { for } Q \in \mathcal{R}_{n+1}(N) \text { with } \operatorname{spt} Q \subset \bar{G} \text { and } \mathbf{M}(\partial Q) \leq s .
$$

For the $H$ volume this implies

$$
\begin{gathered}
\left|\mathbf{V}_{H}\left(T, T_{0}\right)\right| \leq c \mathbf{M}\left(T-T_{0}\right) \\
\text { if } T \in \mathcal{C}\left(T_{0}, G, \sigma\right) \text { and }(1+\sigma) \mathbf{M}\left(T_{0}\right) \leq s .
\end{gathered}
$$

All the results of this section are based on the following theorem. We exclude the case $T_{0}=0$ there, because then $T_{0}$ itself is a minimizer in (i), and in fact the only one if $c<1$. Note, however, that we may have $\partial T_{0}=0, T_{0} \neq 0$; the theorem then gives $H$ energy minimizing closed currents $T$ in the homology class of $T_{0}$, if the hypotheses are satisfied.

3.2. Theorem. Suppose $0 \neq T_{0} \in \mathcal{R}_{n}(N)$, spt $T_{0} \subset \bar{G}, 1<\sigma \leq \infty$, $(1+\sigma) \mathbf{M}\left(T_{0}\right) \leq s$, the isoperimetric constant $c_{G}(s)$ is finite and $H$ satisfies an isoperimetric condition of type $(c, s)$ on $\bar{G}$ where $c \leq 1$ and $c<1$ if $\sigma=\infty$. Then:

(i) there exists a minimizer $T$ of the $H$ energy in $\mathcal{C}\left(T_{0}, G, \sigma\right)$; 
(ii) each minimizer $T$ with $\mathbf{M}(T)<\sigma \mathbf{M}\left(T_{0}\right)$ (no condition, if $\sigma=\infty$ ) is a current of prescribed mean curvature $H$ in $N$, provided $|H|$ does not exceed the inward mean curvature $H_{G}$ along $\partial G($ no condition if $\partial G=\varnothing)$;

(iii) if, in addition to the conditions in (ii), $|H|<H_{G}$ holds along $\partial G$, then we have $\partial G \cap \operatorname{spt} T \backslash$ spt $\partial T_{0}=\varnothing$.

Proof. Since (ii), (iii) follow immediately from Lemma 1.3 and Lemma 1.5 it is sufficient to prove (i). We consider a minimizing sequence $T_{k}$ for $\mathbf{E}_{H}$ on $\mathcal{C}\left(T_{0}, G, \sigma\right)$. From (3) we get

$$
\begin{aligned}
\mathbf{E}_{H}\left(T_{k}\right) & \geq(1-c) \mathbf{M}\left(T_{k}\right)-c \mathbf{M}\left(T_{0}\right)+c \mathbf{M}\left(T_{k}-T_{0}\right)-\left|\mathbf{V}_{H}\left(T_{k}, T_{0}\right)\right| \\
& \geq(1-c) \mathbf{M}\left(T_{k}\right)-c \mathbf{M}\left(T_{0}\right) .
\end{aligned}
$$

Therefore $\mathbf{E}_{H}\left(T_{k}\right)$ is bounded from below and, as $c<1$ is assumed in the case $\sigma=\infty$, the masses $\mathbf{M}\left(T_{k}\right)$ are bounded from above. Let $Q_{k} \in \mathcal{R}_{n+1}(N)$ be the currents with spt $Q_{k} \subset \bar{G}$, and $\partial Q_{k}=T_{k}-T_{0}$. Since, by hypothesis, $\mathbf{M}\left(\partial Q_{k}\right) \leq \mathbf{M}\left(T_{k}\right)+\mathbf{M}\left(T_{0}\right) \leq(1+\sigma) \mathbf{M}\left(T_{0}\right) \leq s$ and $c_{G}(s)<\infty$, we also have a mass bound

$$
\sup _{k} \mathbf{M}\left(Q_{k}\right) \leq c_{G}(s) \sup _{k} \mathbf{M}\left(\partial Q_{k}\right)=\mu<\infty
$$

(cf. Definition 2.1). It follows from the $B V$ compactness theorem [EG, 5.2], $[\mathrm{Zi}, 5.3]$ that the integer valued multiplicity functions $\zeta_{Q_{k}}$, after passage to a subsequence, converge in $L_{\text {loc }}^{1}(N)$ to the multiplicity function $\zeta_{Q}$ of some $Q \in \mathcal{R}_{n+1}(N)$ with spt $Q \subset \bar{G}$ and the $T_{k}$ converge weakly to $T=\partial Q+T_{0} \in$ $\mathcal{C}\left(T_{0}, G, \sigma\right)$. If $\bar{G}$ is compact, then we have $L^{1}$ convergence $\zeta_{Q_{k}} \rightarrow \zeta_{Q}$ and deduce immediately

$$
\begin{aligned}
\mathbf{V}_{H}\left(T, T_{0}\right) & =\int H \zeta_{Q} d \mathcal{H}^{n+1} \\
& =\lim _{k \rightarrow \infty} \int H \zeta_{Q_{k}} d \mathcal{H}^{n+1} \\
& =\lim _{k \rightarrow \infty} \mathbf{V}_{H}\left(T_{k}, T_{0}\right)
\end{aligned}
$$

This implies

$$
\mathbf{E}_{H}(T) \leq \liminf _{k \rightarrow \infty}\left[\mathbf{M}\left(T_{k}\right)+\mathbf{V}_{H}\left(T_{k}, T_{0}\right)\right]=\liminf _{k \rightarrow \infty} \mathbf{E}_{H}\left(T_{k}\right),
$$

by lower semicontinuity of mass, and (i) is proved.

If $\bar{G}$ is not compact then (3.5) is not true, in general, but we can estimate the possible jump of the $H$ volume near infinity by the jump of the mass near infinity (see (3.8)) to prove (3.6). For this we fix a point $a \in N$ and use slicing with the distance to $a$ to find, for a given $R>0$, radii $\left.r_{k} \in\right] R, 2 R[$ such that for the open balls $B_{r_{k}}$ with center $a$ we have

$$
\mathbf{M}\left(\partial \left(Q_{k}\left\llcorner B_{r_{k}}\right)-\left(\partial Q_{k}\right)\left\llcorner B_{r_{k}}\right) \leq \frac{1}{R} \mathbf{M}\left(Q_{k}\left\llcorner\left(B_{2 R} \backslash \overline{B_{R}}\right)\right) \leq \frac{1}{R} \mu .\right.\right.\right.
$$


For the currents $Q_{k}\left\llcorner\left(N \backslash B_{r_{k}}\right)\right.$ this gives the boundary mass estimate

$$
\begin{aligned}
\mathbf{M}\left(\partial \left(Q_{k}\left\llcorner\left(N \backslash B_{r_{k}}\right)\right)\right.\right. & \leq \mathbf{M}\left(\partial Q_{k}\left\llcorner\left(N \backslash B_{r_{k}}\right)\right)+\frac{1}{R} \mu\right. \\
& \leq \mathbf{M}\left(T_{k}\left\llcorner\left(N \backslash B_{r_{k}}\right)\right)+\mathbf{M}\left(T_{0}\right)\right. \\
& <(1+\sigma) \mathbf{M}\left(T_{0}\right) \leq s,
\end{aligned}
$$

provided $R$ is sufficiently large. We use the isoperimetric condition (3.3) with the currents $Q_{k}\left\llcorner\left(N \backslash B_{r_{k}}\right)\right.$ and $c \leq 1$, to infer from (3.7)

$$
\begin{aligned}
\left|\int_{N \backslash B_{r_{k}}} H \zeta_{Q_{k}} d \mathcal{H}^{n+1}\right| \leq \mathbf{M}\left(T_{k}\left\llcorner\left(N \backslash B_{r_{k}}\right)\right)\right. & \\
& +\mathbf{M}\left(T_{0}\left\llcorner\left(N \backslash B_{R}\right)\right)+\frac{1}{R} \mu .\right.
\end{aligned}
$$

We also note

$$
\lim _{k \rightarrow \infty} \int_{B_{r_{k}}}\left(H \zeta_{Q_{k}}-H \zeta_{Q}\right) d \mathcal{H}^{n+1}=0,
$$

because $\zeta_{Q_{k}} \rightarrow \zeta_{Q}$ in $L^{1}$ norm on $B_{2 R}$ and $H$ is bounded.

With (3.8) and (3.9) we finally see

$$
\begin{aligned}
& \mathbf{M}\left(T\left\llcorner B_{R}\right)+\mathbf{V}_{H}\left(T, T_{0}\right)\right. \\
= & \mathbf{M}\left(T\left\llcorner B_{R}\right)+\int_{N} H \zeta_{Q} d \mathcal{H}^{n+1}\right. \\
= & \mathbf{M}\left(T\left\llcorner B_{R}\right)+\lim _{k \rightarrow \infty}\left[\int_{N} H \zeta_{Q_{k}} d \mathcal{H}^{n+1}-\int_{N \backslash B_{r_{k}}} H \zeta_{Q_{k}} d \mathcal{H}^{n+1}\right.\right. \\
& \left.-\int_{N \backslash B_{r_{k}}} H \zeta_{Q} d \mathcal{H}^{n+1}\right] \\
\leq & \liminf _{k \rightarrow \infty}\left[\mathbf{M}\left(T_{k}\right)+\mathbf{V}_{H}\left(T_{k}, T_{0}\right)+\mathbf{M}\left(T_{0}\left\llcorner\left(N \backslash B_{R}\right)\right)+\frac{1}{R} \mu\right.\right. \\
& \left.+\int_{N \backslash B_{R}}\left|H \zeta_{Q}\right| d \mathcal{H}^{n+1}\right],
\end{aligned}
$$

and letting $R \rightarrow \infty$, we deduce (3.6) again.

3.3. Remarks. The minimization process used above implies special properties for the resulting $\mathbf{E}_{H}$ minimizing currents $T$ with prescribed mean curvature $H$, which we find in Theorem 3.2 (ii):

(1) $T$ is $\lambda$ minimizing in $\bar{G}$ for $\lambda=c_{G}(s) \sup |H|<\infty$, i.e. $\mathbf{M}(T) \leq$ $\mathbf{M}(T+\partial Q)+\lambda \mathbf{M}(Q)$ holds for all $Q \in \mathcal{R}_{n+1}(N)$ with $\operatorname{spt} Q \subset \bar{G}$. This follows from $\mathbf{E}_{H}(T) \leq \mathbf{E}_{H}(T+\partial Q)$ and (2.18) in the case $\mathbf{M}(T+\partial Q) \leq \sigma \mathbf{M}\left(T_{0}\right)$ 
and is trivial otherwise. See [DS1], [DS2] for the notion of $\lambda$ minimality and its consequences in Euclidean space $\mathbb{R}^{n+1}$.

(2) T satisfies a monotonicity formula as in [Al], [Si], [Du], [DS1], [DS2]. As a consequence, the $n$ dimensional density of $\|T\|$ exists everywhere and is upper semicontinuous on spt $T \backslash$ spt $\partial T$. Furthermore, spt $T$ is compact if spt $\partial T$ is compact, and otherwise $\operatorname{dist}(x, \operatorname{spt} \partial T) \rightarrow 0$ as $x$ tends to infinity in spt $T$.

(3) If $c<1$, then $T$ is indecomposable in the sense that $T=T^{\prime}+T^{\prime \prime}$ with $T^{\prime} \in \mathcal{C}\left(T_{0}, G\right), \partial T^{\prime \prime}=0$, and $\mathbf{M}(T)=\mathbf{M}\left(T^{\prime}\right)+\mathbf{M}\left(T^{\prime \prime}\right)$ implies $T^{\prime \prime}=0$. This is immediate from $\mathbf{E}_{H}\left(T^{\prime}\right) \geq \mathbf{E}_{H}(T)$ and (3). (Note, however, that such decompositions will exist, in general, with $T^{\prime}$ not homologous to $T$ in $\bar{G}$.) As a consequence, spt $T$ is connected if spt $\partial T$ is connected and if every closed current in $\mathcal{R}_{n}(N)$ with support in $\bar{G}$ is homologous to zero in $\bar{G}$.

(4) If $H$ is locally Lipschitz, then one has an interior regularity theory asserting that $G \cap \operatorname{spt} T \backslash$ spt $\partial T$ is the union of a submanifold of class $C^{2, \beta}$, on which $T$ has constant multiplicity, and a possible singular set, which is of Hausdorff dimension at most $n-7$ (and discret for $n=7$, empty for $n<7$ ). Furthermore, one has complete boundary regularity (no singularities on or close to the boundary) near points on $G \cap$ spt $\partial T$, where $\partial T=\partial T_{0}$ is represented by an oriented smooth submanifold of dimension $n-1$ with constant integer multiplicity. This has been proved for the Euclidean case $N=\mathbb{R}^{n+1}$ in [DS1], [DS2] and, as pointed out in $[\mathrm{HSi}]$, the proofs remain valid if the Euclidean mass is replaced by an ellipsoidal integrand (mass with respect to some Riemannian metric, which can be assumed arbitrarily close to the Euclidean metric for purposes of regularity theory).

There are various ways to deduce an isoperimetric condition for $H$, as required in the previous theorem, from the isoperimetric inequalities treated in Section 2. We present some variants in

3.4. Proposition. An isoperimetric condition of type $(1, s)$ holds for $H$ on $\bar{G}$ if one of the following conditions is satisfied:

(i) An isoperimetric inequality $\mathcal{H}^{n+1}(A) \leq c \mathbf{M}(\partial \llbracket A \rrbracket)$ with $0 \leq c<\infty$ is valid for sets of finite perimeter $A \subset G$ with $\mathbf{M}(\partial \llbracket A \rrbracket) \leq s$, and $\sup _{G}|H| \leq c^{-1}$;

(ii) An isoperimetric inequality $\mathcal{H}^{n+1}(A) \leq \gamma \mathbf{M}(\partial \llbracket A \rrbracket)^{1+1 / n}$ is valid for sets of finite perimeter $A \subset G$ with $\mathbf{M}(\partial \llbracket A \rrbracket) \leq s$, and $\int_{G}|H|^{n+1} d \mathcal{H}^{n+1} \leq \gamma^{-n}$;

(iii) We have $b\left(\mathcal{H}^{n+1}(A)\right) \leq \mathbf{M}(\partial \llbracket A \rrbracket)$ for sets of finite perimeter $\bar{A} \subset G$ with $\mathbf{M}(\partial \llbracket A \rrbracket) \leq s$, and $\int_{G} a(|H(x)|) d \mathcal{H}^{n+1} \leq 1$ holds for some convex, strictly increasing, nonnegative function a on $\left[0, \infty\left[\right.\right.$ with $t^{-1}(1 / t) \leq b(t)$ for $0<t<\infty$;

(iv) $s=\infty, b$ is as in (iii), and $\mathcal{H}^{n+1}([|H|>t]) \leq \mathcal{H}^{1}([h>t])$ holds for $t>0$ and some nonnegative and nonincreasing function $h$ on $] 0, \infty[$ with $\int_{0}^{u} h(t) d t \leq b(u)$ for $u>0$; 
(v) $s=\infty$, the mean curvature function $H_{G}$ of $G$ (see Lemma 1.5) is nonnegative, and $|H| \leq H_{G}$ on $G \mathcal{H}^{n+1}$ almost everywhere;

(vi) $s=\infty, N=N^{\prime} \times N^{\prime \prime}$ is a product of Riemannian manifolds, $G=G^{\prime} \times G^{\prime \prime}$ a product of domains $G^{\prime} \subset N^{\prime}$ and $G^{\prime \prime} \subset N^{\prime \prime}$, and $x^{\prime} \mapsto H\left(x^{\prime}, x^{\prime \prime}\right)$ satisfies an isoperimetric condition of type $(1, \infty)$ on $\overline{G^{\prime}}$ for all $x^{\prime \prime} \in G^{\prime \prime}$.

Proof. From $\left|\int_{A} H d \mathcal{H}^{n+1}\right| \leq \mathcal{H}^{n+1}(A) \sup |H|$ and from Hölder's inequality we get (i) and (ii). (iii) was proved in Section 2 (see (2.14) and the subsequent discussion). To prove (iv) we note

$$
\begin{aligned}
\int_{A}|H| d \mathcal{H}^{n+1} & =\int_{0}^{\infty} \mathcal{H}^{n+1}(A \cap[|H|>t]) d t \\
& \leq \int_{0}^{\infty} \min \left\{\mathcal{H}^{n+1}(A), \mathcal{H}^{n+1}([|H|>t])\right\} d t \\
& \leq \min \left\{\mathcal{H}^{n+1}(A), \mathcal{H}^{1}([|h|>t])\right\} d t \\
& =\int_{0}^{\mathcal{H}^{n+1}(A)} h(t) d t \leq b\left(\mathcal{H}^{n+1}(A)\right) \leq \mathbf{M}(\partial \llbracket A \rrbracket) .
\end{aligned}
$$

(v) was also proved in Section 2, see Remark 2.10. Finally, (vi) follows from the coarea formula applied to the projection $G=G^{\prime} \times G^{\prime \prime} \rightarrow G^{\prime \prime}$.

It is clear that (vi) holds more generally if $G_{x^{\prime \prime}}:=\left\{x^{\prime} \in N^{\prime}:\left(x^{\prime}, x^{\prime \prime}\right) \in G\right\}$ depends on $x^{\prime \prime}$ and $H\left(\cdot, x^{\prime \prime}\right)$ satisfies an isoperimetric condition of type $(1, \infty)$ on $\bar{G}_{x^{\prime \prime}}$ for all $x^{\prime \prime} \in G^{\prime \prime}$. One may also consider more general metrics on $N=$ $N^{\prime} \times N^{\prime \prime}$ than the product metric. In general, isoperimetric conditions will be preserved, with a change of the type $(c, s)$, if one changes the Riemannian metric to a new metric which is biLipschitz equivalent on $\bar{G}$ to the original one. We also note that one may combine various sufficient conditions like (i)-(vi) above, because the set of functions $H$ satisfying an isoperimetric condition of fixed type on $\bar{G}$ is convex. Furthermore, (ii)-(iv) can be improved with the following observation:

3.5. Remark. If $H=H_{+}-H_{-}$is decomposed into its positive and negative parts and $H_{+}, H_{-}$both satisfy an isoperimetric condition of type $(c, s)$ on $\bar{G}$, then also $H$ satisfies this isoperimetric condition. This is an immediate consequence of the inequality $\left|\int_{A} H d \mathcal{H}^{n+1}\right| \leq \max \left\{\int_{A} H_{+} d \mathcal{H}^{n+1}, \int_{A} H_{-} d \mathcal{H}^{n+1}\right\}$.

We now formulate some more explicit sufficient geometric conditions for the existence of currents with prescribed mean curvature.

\subsection{Theorem.}

(i) If the isoperimetric constant $c_{N}(\infty)$ is finite and $H: N \rightarrow \mathbb{R}$ is continuous with $\sup _{N}|H|<c_{N}(\infty)^{-1}$, then for any $T_{0} \in \mathcal{R}_{n}(N)$ there exists a current $T \in \mathcal{R}_{n}(N)$ with prescribed mean curvature $H$ and $\partial T=\partial T_{0}$. 
(ii) If $\bar{G} \neq N, c_{G}(\infty)<\infty, H: \bar{G} \rightarrow \mathbb{R}$ is continuous with $\sup _{G}|H|<$ $c_{G}(\infty)^{-1}$ and $|H|$ does not exceed on $\partial G$ the (inward) mean curvature of $\partial G$, then for any $T_{0} \in \mathcal{R}_{n}(N)$ with spt $T_{0} \subset \bar{G}$ there exists a current $T \in \mathcal{R}_{n}(N)$ with prescribed mean curvature $H$ and homologous to $T_{0}$ in $\bar{G}$.

The proof follows readily from Theorem 3.2 and Proposition 3.4 (i). Part (i) of the theorem can be applied to simply connected manifolds $N$ with negative sectional curvature bounded from above by a negative constant (Corollary 2.7). With regard to part (ii) we note that $c_{\bar{G}}(\infty)<\infty$ holds if $\bar{G} \neq N$ is compact (by Remark $2.4(2)$ ). Thus, we have the two corollaries:

3.7. Corollary. If $N$ is simply connected with sectional curvatures not exceeding $\kappa^{2}<0$ and if $\sup _{N}|H|<n|\kappa|$, then for any $T_{0} \in \mathcal{R}_{n}(N)$ there exists a current $T \in \mathcal{R}_{n}(N)$ with prescribed mean curvature $H$ and $\partial T=\partial T_{0}$.

In the context of two dimensional parametric surfaces of the type of the disc in 3 manifolds this was proved by Gulliver [Gu1], and his method yields also Corollary 3.7.

3.8. Corollary. If $\bar{G} \neq N$ is compact and the (inward) mean curvature of $\partial G$ is positive, then there exists $h(G)>0$ such that for $\sup _{G}|H|<h(G)$ and $T_{0} \in \mathcal{R}_{n}(N)$ with spt $T_{0} \subset \bar{G}$ we have existence of a current $T \in \mathcal{R}_{n}(N)$ with prescribed mean curvature $H$ and homologous to $T_{0}$ in $\bar{G}$.

It is clear from the reasoning leading to this result that the constant $h(G)$ can be estimated from below in terms of the boundary mean curvature of $\partial G$, the volume of $G$, and bounds for the sectional curvatures and the injectivity radius on $\bar{G}$. Note that $G$ need not be homologically trivial. It is easy to find examples of manifolds $N$ and closed nontrivial currents $T_{0} \in \mathcal{R}_{n}(N)$ that are homologically mass minimizing such that there exists a relatively compact domain $G$ on $N$ with positive inward mean boundary curvature and $\operatorname{spt} T_{0} \subset G$. By the corollary we can then find, for sufficiently small $|H|, H$ energy minimizing closed currents $T$ in the homology class of $T_{0}$. Another consequence of Corollary 3.8 is the following: If it is known that there exists no current $T \in \mathcal{R}_{n}(N)$ of constant mean curvature different from zero which is homologous to $T_{0}$, then spt $T_{0}$ cannot be contained in a relatively compact proper subdomain of $N$ with positive inward mean boundary curvature. This observation applies e.g. to a great half $n$ sphere in $N=S^{n+1}$ and to $T_{0}=\llbracket M \times\{t\} \rrbracket$ in $N=M \times \mathbb{R}$ or in $N=M \times S^{1}$ (see Remark 1.4).

In the case $c_{G}(\infty)<\infty$ we can use all the sufficient conditions from Proposition 3.4 to prove existence with conditions on $H$ not requiring smallness of $\sup |H|$. To formulate some of these results we assume that an inequality $\mathcal{H}^{n+1}(A) \leq \gamma \mathbf{M}(\partial \llbracket A \rrbracket)^{1+1 / n}$ is valid for sets $A \subset G$ of finite perimeter. Note that such an inequality with a constant $\gamma<\infty$ follows from $c_{G}(\infty)<\infty$ 
and from Corollary 2.3 whenever $\bar{G}$ is compact or $N$ is homogeneously regular. The constant $\gamma$ is not smaller than the isoperimetric constant $\gamma_{n+1}=$ $\left((1 /(n+1)) \omega(n)^{-1 / n}\right.$ of $\mathbb{R}^{n+1}$ and can be explicitely determined or estimated from above in many interesting cases (cf. Remark 2.4, Example 2.5, Remark 2.8 $(3))$.

3.9. Theorem. Suppose $G \subset N$ is such that $c=c_{G}(\infty)<\infty$ and $\mathcal{H}^{n+1}(A)$

$\leq \gamma \mathbf{M}(\partial \llbracket A \rrbracket)^{(n+1) / n}$ holds for all sets $A \subset G$ with finite perimeter. In case $G \neq N$ suppose further that $|H|$ does not exceed on $\partial G$ the inward mean curvature of $\partial G$. Then, for any given $T_{0} \in \mathcal{R}_{n}(N)$ with $\operatorname{spt} T_{0} \subset \bar{G}$, a current $T \in \mathcal{R}_{n}(N)$ with prescribed mean curvature $H$ and homologous to $T_{0}$ in $\bar{G}$ exists, provided $H$ satisfies one of the following conditions:

$$
\begin{gathered}
\int_{G}|H|^{n+1} d \mathcal{H}^{n+1}<\gamma^{-n} ; \\
\int_{G \cap[c|H|>n /(n+1)]}|H|^{n+1} d \mathcal{H}^{n+1}<\gamma^{-n} ; \\
\sup _{[t>u]} t^{-n-1} \mathcal{H}^{n+1}\{x \in G:|H(x)|>t\}<\left(\frac{n}{n+1}\right)^{1 /(n+1)} \gamma^{-n} \\
\text { for some } u<\frac{n}{n+1} \frac{1}{c} .
\end{gathered}
$$

Proof. (i) follows from Theorem 3.2 and Proposition 3.4 (ii). For (ii) one uses part (iii) of this proposition with

$$
b(t)=\max \left\{\frac{t}{c},\left(\frac{t}{\gamma}\right)^{n /(n+1)}\right\}
$$

and $a(t)$ the largest nonnegative convex function with $a(t)=\gamma^{n} t^{n+1}$ for $t \geq c^{-1}$ (see the discussion after (2.16)). For (iii) we apply Proposition 3.4 (iv) with

$$
h(t)=\frac{n}{n+1} \min \left\{\frac{1}{c}, \gamma^{(-n /(n+1))} t^{(-1 /(n+1))}\right\} .
$$


We remark that it suffices to satisfy the inequalities for $H_{+}$and $H_{-}$instead of $|H|$ (Remark 3.5), and with weak inequalities instead of strict ones if $\bar{G}$ is compact. For (i) the condition $c_{G}(\infty)<\infty$ is not really necessary; in the proof of Theorem 3.2 one can then use boundedness and weak subconvergence of the multiplicity functions $\zeta_{Q_{k}}$ in $L_{\text {loc }}^{1+1 / n}(N)$ in order to prove continuity of the volume $\left\langle Q_{k}, H \Omega\right\rangle \rightarrow\langle Q, H \Omega\rangle$ as $k \rightarrow \infty$ (the limit current will then satisfy $\int\left|\zeta_{Q}\right|^{n+1} d \mathcal{H}^{n+1}<\infty$, but its mass may be infinite). Thus, (ii) can be considered as a sharpening of (i), which is valid when not only $\mathcal{H}^{n+1}(A)$ $\leq \gamma \mathbf{M}(\partial \llbracket A \rrbracket)^{1+1 / n}$ is available (like in the Euclidean space $\mathbb{R}^{n+1}$ ) but also $\mathcal{H}^{n+1}(A) \leq c \mathbf{M}(\partial \llbracket A \rrbracket)$ (like in hyperbolic spaces $N_{\kappa}, \kappa^{2}<0$, for example). Variants and improvements of (ii) can be deduced from Section 2. Further improvements are possible if it is known that the isoperimetric profile $b(t)$ is a concave function. Then the factors $n /(n+1)$ appearing in (ii) and (iii) can be replaced by 1 . In particular, this is the case in spaces $N_{\kappa}$ of constant curvature where also the optimal constants $c, \gamma$ are known explicitely (see Example 2.5). By [Kl] this sharpening of statements (ii) and (iii) is also valid if $\operatorname{dim} N=3$ and $N$ has sectional curvatures not exceeding $\kappa^{2} \leq 0$.

We now specialize to the case where $G$ is a normal neighborhood of a point in a manifold $N$ with upper bound $\kappa^{2}$ for its sectional curvatures. For the functions $\alpha_{\kappa}(r), \beta_{\kappa}(r), c_{\kappa}(r)$ see Example 2.5. We also use

$$
\begin{array}{r}
\gamma_{\kappa}(r):=\gamma_{n+1}=\frac{1}{n+1} \omega(n)^{-1 / n} \text { if } \kappa^{2} \leq 0 \\
\gamma_{\kappa}(r):=\alpha_{\kappa}(r) \beta_{\kappa}(r)^{-1-1 / n} \text { if } \kappa^{2}>0 .
\end{array}
$$

3.10. Theorem. Suppose $N$ has sectional curvatures not exceeding $\kappa^{2}, G$ is a normal neighborhood of $a \in N$ contained in the ball $B_{r}(a)$ with $0<r<\infty$ and $|\kappa| r<\pi$ in the case $\kappa^{2}>0$, the inward mean curvature on $\partial G$ is not smaller than $|H|$ on $\partial G$, and $T_{0} \in \mathcal{R}_{n}(N)$ with spt $T_{0} \subset \bar{G}$. Then a current $T \in \mathcal{R}_{n}(N)$ with prescribed mean curvature $H$ and with $\partial T=\partial T_{0}$ and $\operatorname{spt} T \subset \bar{G}$ exists, provided one of the following conditions is satisfied:

(i) $\sup _{G}|H|<\frac{1}{c_{\kappa}(r)}$;

(ii) there exists $c<1$ such that

$$
|H(x)| \leq c n \kappa \cot \kappa \varrho \text { for } 0<\varrho<r \text { and } x \in G \cap B_{\varrho}(a)
$$

(here we assume $|\kappa| r<\frac{1}{2} \pi$ if $\kappa^{2}>0$ );

(iii) $N$ has constant sectional curvature and

$$
\int_{G \cap\left[c_{\kappa}(r)|H| \geq 1\right]}|H|^{n+1} d \mathcal{H}^{n+1}<\gamma_{\kappa}(r)^{-n} ;
$$


(iv) $N$ has constant sectional curvature and there exists $c<1$ such that

$$
\begin{gathered}
\qquad \mathcal{H}^{n+1}\{x \in G:|H(x)|>c n \kappa \cot \kappa \varrho\} \leq \alpha_{\kappa}(r) \\
\text { for } 0<\varrho<r \text { and } x \in G \cap B_{\varrho}(a) \\
\text { (again we assume } \left.|\kappa| r<\frac{1}{2} \pi \text { if } \kappa^{2}>0\right) .
\end{gathered}
$$

Proof. Pulling back the metric to $\operatorname{Tan}_{a} N$, we may assume that $B_{r}(a)$ is a normal coordinate ball. (i) then follows from Remark 2.8 (2) and Theorem 3.6, while (ii) is a consequence of Theorem 3.2, Proposition $3.4(\mathrm{v})$, Remark $2.10(1)$, and the fact that the inward mean curvature of a sphere $\partial B_{\varrho}(a)$ is at least $n \kappa \cot \kappa \varrho$ (see the proof preceding Proposition 2.6). Statement (iii) follows from Theorem 3.9 and the improvements noted thereafter. Finally, for (iv) we use the explicit form of the isoperimetric profile $b_{\kappa}(t)$ for $N_{\kappa}$ determined in Example 2.5 to see that Proposition 3.4 (iv) can be applied with $h(t)=$ $n \kappa \cot \left(\kappa \alpha_{\kappa}^{-1}(t)\right)$.

Again, it suffices to require the conditions in (iii) and (iv) for $H_{+}, H_{-}$instead of $H$, and the constancy of curvature is not needed in the case $\operatorname{dim} N=3$ and $\kappa^{2} \leq 0$, by [Kl]. For parametric surfaces in a 3 manifold (i) has been proved in $[\mathrm{HiK}],[\mathrm{Gu} 1]$, with stronger hypotheses, however. For example, in the case $G=B_{r}(a)$ the constant $n \kappa \cot \kappa r<c_{\kappa}(r)^{-1}$ was used in [Gu1] (in particular, $n \kappa \cot \kappa r=n / r$ and $c_{\kappa}(r)^{-1}=(n+1) / r$ in the case $\left.\kappa^{2}=0\right)$. Note that $n \kappa \cot \kappa r$ is a lower bound for the mean curvature of $\partial B_{r}(a)$. Thus, our result (i) above is an improvement in two respects: On the boundary instead of $|H| \leq n \kappa \cot \kappa r$ we require only that $|H|$ do not exceed the mean curvature of $\partial B_{r}(a)$, and in the interior we allow the larger value $c_{\kappa}(r)^{-1}$ for $\sup |H|$. For constant prescribed mean curvature and constant curvature spaces $N_{\kappa}$ the result in [HiK], [Gu1] is the same as part (i) of the preceding theorem and it is optimal (cf. Remark 1.4). We note that conditions (i)-(iv) above are optimal for $N_{\kappa}$ in the sense that the infimum of the $H$ energy in the classes in which we minimize can be $-\infty$, if the constants are replaced by larger ones. In this sense the conditions are necessary for the existence of $\mathbf{E}_{H}$ minimizing currents.

We now turn to the case $c_{N}(\infty)=\infty$, i.e. no inequality $\mathcal{H}^{n+1}(A)<c \mathbf{M}(\partial \llbracket A \rrbracket)$ is valid for all sets $A \subset N$ with finite perimeter. Sometimes we can reduce the problem of the existence of a current $T \in \mathcal{R}_{n}(N)$ with prescribed mean curvature $H$ and boundary $\partial T=\partial T_{0}$ to a situation where one has such an unrestricted linear isoperimetric inequality. For example, if $N$ has sectional curvatures $\leq \kappa^{2}<0$, and $T_{0}$ can be lifted to the universal covering $\tilde{N}$ of $N$, then we can solve the problem working in $\tilde{N}$ (cf. [Gu1, Sec. 6] for arguments of this sort). In general, however, such a reduction will not be possible, and for the isoperimetric function we only know $c_{N}(s)<\infty$ for some finite number $s$. We then have to use Theorem 3.2 with a constraint $\mathbf{M}(T) \leq \sigma \mathbf{M}\left(T_{0}\right)<\infty$, and we must verify strict inequality for the minimizer. It has been shown by Wente [We], how this can be done in the setting of parametric surfaces in $\mathbb{R}^{3}$, and the same idea works here too: 
3.11. Theorem. Suppose $0<s<\infty, c_{G}(s)<\infty, T_{0} \in \mathcal{R}_{n}(N)$ with spt $T_{0} \subset \bar{G}$ and $2 \mathbf{M}\left(T_{0}\right)<s$, and $|H|$ does not exceed on $\partial G$ the inward mean curvature of $\partial G$ (if $G \neq N$ ). Then there exists a current $T \in \mathcal{R}_{n}(N)$ of prescribed mean curvature $H$ homologous to $T_{0}$ in $\bar{G}$, provided

$$
\sup _{G}|H|<\frac{s-2 \mathbf{M}\left(T_{0}\right)}{s c_{G}(s)} .
$$

Proof. An isoperimetric condition (3.2) of type $(c, s)$ is valid with $c=$ $c_{G}(s) \sup |H|$. We want to apply Theorem 3.2 (i) with $\sigma>1,(1+\sigma) \mathbf{M}\left(T_{0}\right) \leq s$; as it turns out, the best choice is $\sigma=s \mathbf{M}\left(T_{0}\right)^{-1}-1$. Since we have, by (3.10),

$$
\sup |H| \leq \frac{1}{c_{G}(s)}
$$

an isoperimetric condition of type $(1, s)$ is satisfied for $H$ on $\bar{G}$, hence we obtain a minimizer $T$ of $\mathbf{E}_{H}$ in $\mathcal{C}\left(T_{0}, G, \sigma\right)$. Comparing $\mathbf{E}_{H}(T)$ with $\mathbf{E}_{H}\left(T_{0}\right)$ and using (3.4), we see, taking also $\mathbf{M}(T) \leq \sigma \mathbf{M}\left(T_{0}\right)$ into account,

$$
\begin{aligned}
\mathbf{M}(T) & =\mathbf{E}_{H}(T)-\mathbf{V}_{H}\left(T, T_{0}\right) \leq \mathbf{E}_{H}\left(T_{0}\right)-\mathbf{V}_{H}\left(T, T_{0}\right) \\
& =\mathbf{M}\left(T_{0}\right)-\mathbf{V}_{H}\left(T, T_{0}\right) \leq \mathbf{M}\left(T_{0}\right)+c \mathbf{M}\left(T-T_{0}\right) \\
& \leq \mathbf{M}\left(T_{0}\right)\left[1+(1+\sigma) c_{G}(s) \sup |H|\right] .
\end{aligned}
$$

The inequality $\mathbf{M}(T)<\sigma \mathbf{M}\left(T_{0}\right)$ now follows from the choice of $\sigma$ and from (3.10), (3.12), hence Theorem 3.2 (ii) can be applied.

It is clear from the proof that we can formulate similar results with other types of hypotheses on $H$ that ensure the validity of an isoperimetric condition of type $(c, s)$ with constant $c$ satisfying $s-2 \mathbf{M}\left(T_{0}\right)>s c$ (cf. Proposition 3.4). The preceding theorem is optimal with regard to the conditions on $T_{0}$; it is, however, most likely not optimal with respect to the condition (3.10) which restricts the size of the admitted mean curvature functions. This can be seen from the examples presented now.

3.12. Examples. (1) We consider $N=M \times \mathbb{R}$ with a closed manifold $M$ as in Proposition 2.11. There it was proved that $c_{N}(s)$ is finite if and only if $s<$ $2 \mathcal{H}^{n}(M)$. Hence, Theorem 3.11 can be applied if and only if $\mathbf{M}\left(T_{0}\right)<\mathcal{H}^{n}(M)$, to establish the existence of currents $T$ homologous to $T_{0}$ with sufficiently small prescribed mean curvature. On the other hand, for the current $T_{0}=\llbracket M \times\{0\} \rrbracket$ with $\mathbf{M}\left(T_{0}\right)=\mathcal{H}^{n}(M)$ we know from Remark 1.4 that no current $T$ with nonvanishing constant mean curvature exists in $N$ which is homologous to $T_{0}$.

The mean curvature functions $H$ allowed by condition (3.10) must be uniformly small if $\mathbf{M}\left(T_{0}\right)$ is close to (and smaller than) $\mathcal{H}^{n}(M)$, because in the limit $s \uparrow 2 \mathcal{H}^{n}(M)$ the denominator in (3.10) stays bounded, by Proposition 2.11. One might suspect that condition (3.10) here is more restrictive than 
necessary. For example, in the case $M=S^{1}$, we can see, by passing to the universal covering, that actually $\sup |H|<1 / \pi$ is sufficient for the existence of a 1 current $T$ in $S^{1} \times \mathbb{R}$ with prescribed curvature $H$ and homologous to a given $T_{0}$ with $\mathbf{M}\left(T_{0}\right)<2 \pi$.

(2) The restriction (3.10) for the mean curvature function is, of course, weakest if we choose $T_{0}$ absolutely mass minimizing in its homology class (this is possible, since the theorem can be applied with $H \equiv 0$ and produces energy minimizing currents) and then maximize the right-hand side in (3.10) on the interval of parameters $s$ with $s>2 \mathbf{M}\left(T_{0}\right)$ and $c_{G}(s)<\infty$ (by hypothesis this interval is nonempty). In the Euclidean case $G=N=\mathbb{R}^{n+1}$, we have $c_{G}(s)=$ $(1 /(n+1)) \omega(n)^{-1 / n} s^{1 / n}$, and the maximum $n \alpha(n+1)^{1 / n} 2^{-1 / n} \mathbf{M}\left(T_{0}\right)^{-1 / n}$ is achieved for $s=2(n+1) \mathbf{M}\left(T_{0}\right)$. (Theorem 3.11 for $N=\mathbb{R}^{n+1}$ and this choice of $s$ was first proved in [Du].) It is conjectured that this maximal value is by a factor

$$
\left(\frac{\alpha(n+1)}{2 \alpha(n)}\right)^{1 / n}<1
$$

smaller than necessary, i.e. one expects existence of a current $T$ with prescribed mean curvature $H$ and boundary $\partial T_{0}$ in $\mathbb{R}^{n+1}$ whenever sup $|H| \leq$ $n \alpha(n)^{1 / n} \mathbf{M}\left(T_{0}\right)^{-1 / n}$. Such a result would be optimal as is shown by $T_{0}$ representing an oriented $n$ disc in $\mathbb{R}^{n+1}$ (cf. Remark 1.4).

(3) A similar, though less explicit, discussion is possible for the standard spaces $N_{\kappa}$ of constant sectional curvature $\kappa^{2}$. Here we consider $\kappa^{2}=-1$; for the case $\kappa^{2}>0$ we refer to Example 4.4. We use the functions $\alpha_{\kappa}(\varrho), \beta_{\kappa}(\varrho)$ from Example 2.5 with $\kappa=\sqrt{-1}$. Substituting $\beta_{\kappa}(\varrho)$ for $s$ in $(3.10)$, we have

$$
\frac{s-2 \mathbf{M}\left(T_{0}\right)}{s c_{N}(s)}=\frac{\beta_{\kappa}(\varrho)-2 \mathbf{M}\left(T_{0}\right)}{\alpha_{\kappa}(\varrho)},
$$

and we check that the derivative with respect to $\varrho$ equals $\alpha_{\kappa}(\varrho)^{-2} \beta_{\kappa}(\varrho) \eta(\varrho)$, where $\eta(\varrho)=\alpha_{\kappa}(\varrho) n \cot \varrho-\beta_{\kappa}(\varrho)+2 \mathbf{M}\left(T_{0}\right)$ is strictly decreasing with $\eta(0)>0$. Therefore, we conclude that either a unique maximum point $r$ exists in the interval $] \beta_{\kappa}^{-1}\left(2 \mathbf{M}\left(T_{0}\right)\right), \infty[$ for the function $(3.13)$ of $\varrho$, and then

$$
\sup |H|<n \operatorname{coth} r
$$

is the weakest possible restriction on $H$ allowed by Theorem 3.11, or the function in (3.13) is strictly increasing in $\varrho$ achieving its supremum $n$ formally at $r=$ $\infty$. The latter case occurs only when $n=1$ and $\mathbf{M}\left(T_{0}\right) \geq \pi$, whereas $r=$ $2 \operatorname{Artanh}\left(\mathbf{M}\left(T_{0}\right) / \pi\right)$ if $n=1$ and $\mathbf{M}\left(T_{0}\right)<\pi$. Since $n \operatorname{coth} r>n$ in any case, we have, in spaces of constant negative sectional curvature, an improvement of Corollary 3.7. However, for $n \geq 2$ this can not easily be made more explicit and Theorem 3.10 will give a better result in many cases. For $n=2$ we find that $r$ is determined by the equation $r \operatorname{coth} r-1=1 / 2 \pi \mathbf{M}\left(T_{0}\right)$.

One of the consequences of Theorem 3.11, which does not follow from the results preceding it, is the following: A given $n-1$ boundary spans an $n$ current 
with large prescribed mean curvature in $N$ if it bounds a current $T_{0}$ of sufficiently small mass, regardless of the diameter or other geometric characteristics of spt $\partial T_{0}$. To give a more precise formulation we assume an isoperimetric inequality $\mathcal{H}^{n+1}(A) \leq \gamma \mathbf{M}(\partial \llbracket A \rrbracket)^{1+1 / n}$ for $A \subset G$ with $\mathbf{M}(\partial \llbracket A \rrbracket) \leq s$. Note that such an inequality follows from Corollary 2.3 if $c_{G}(s)<\infty$ and $\bar{G}$ is compact or $N$ is homogeneously regular. We then have the following result:

3.13. Corollary. Suppose $0<s_{0} \leq \infty$, an inequality

$$
\mathcal{H}^{n+1}(A) \leq \gamma \mathbf{M}(\partial \llbracket A \rrbracket)^{1+1 / n}
$$

is valid for sets $A$ of finite perimeter in $G$ with $\mathbf{M}(\partial \llbracket A \rrbracket) \leq s_{0}$, and $T_{0} \in \mathcal{R}_{n}(N)$ with $\operatorname{spt} T_{0} \subset \bar{G}$ and $2(n+1) \mathbf{M}\left(T_{0}\right) \leq s_{0}$. Then there exists a current $T$ of prescribed mean curvature $H$ and homologous to $T_{0}$ in $\bar{G}$, whenever

$$
\sup _{G}|H|<\frac{n}{(n+1) \gamma\left[2(n+1) \mathbf{M}\left(T_{0}\right)\right]^{1 / n}}
$$

and $|H|$ does not exceed on $\partial G$ the inward mean boundary curvature of $\partial G$ (if $G \neq N)$.

Proof. We observe that the isoperimetric inequality in the hypothesis implies $c_{G}(s) \leq \gamma s^{1 / n}$ for $0 \leq s \leq s_{0}$. Applying Theorem 3.11 with $2 \mathbf{M}\left(T_{0}\right)<s \leq s_{0}$, we therefore deduce the existence of $T$ with the asserted properties, provided we have the inequality

$$
\sup _{G}|H|<\frac{s-2 \mathbf{M}\left(T_{0}\right)}{\gamma s^{1+1 / n}} .
$$

With the optimal choice $s=2(n+1) \mathbf{M}\left(T_{0}\right)$, this is just (3.14).

Since $\gamma$ must be at least as large as the corresponding isoperimetric constant of Euclidean space $\mathbb{R}^{n+1}$, the bounds for the admissible mean curvatures $H$ determined by (3.14) can never be better than the corresponding bounds in $\mathbb{R}^{n+1}$.

We have a further existence result for currents $T$ of prescribed mean curvature homologous to $T_{0}$ in a non-closed manifold $N$. In this result we assume as hypothesis that $T_{0}$ is uniquely mass minimizing in its homology class (a somewhat weaker condition is actually sufficient). Since the proof can be better understood after the discussion of the corresponding result for closed manifolds $N$, we postpone the statement of this result to the end of the next section (Theorem 4.7).

4. Existence in closed manifolds. The general assumption for this section is that the $n+1$ dimensional, oriented and connected Riemannian manifold $N$ is closed, i.e. compact without boundary. We could also treat complete Riemannian manifolds with finite volume in which the relevant isoperimetric 
inequalities are satisfied. However, we will not consider this more general situation in the sequel. The prescribed mean curvature function $H: N \rightarrow \mathbb{R}$ will be assumed continuous, although less restrictive conditions would suffice.

In trying to carry over the approach from Section 3 to a closed manifold $N$ we face the following problem: If $T$ and $T_{0}$ are two homologous integer multiplicity rectifiable $n$ currents in $N$, then the current $Q \in \mathcal{R}_{n+1}(N)$ with $\partial Q=T-T_{0}$ is not unique. In fact, $Q$ is determined only up to integer multiples of $\llbracket N \rrbracket$, by the constancy theorem. Therefore, the definition of volume

$$
\mathbf{V}_{H}\left(T, T_{0}\right)=\langle Q, H \Omega\rangle=\int_{N} \zeta_{Q} H d \mathcal{H}^{n+1} \quad \text { when } \partial Q=T-T_{0}
$$

( $\Omega$ the volume form of $N, \zeta_{Q}$ the integer valued multiplicity function of $Q$ ) is no longer possible; $\mathbf{V}_{H}\left(T, T_{0}\right)$ would only be determined up to integer multiples of $\langle\llbracket N \rrbracket, H \Omega\rangle=\int_{N} H d \mathcal{H}^{n+1}$. There is an obvious way to avoid this problem. Namely, one could require the vanishing of the mean value of $H$ on $N$. (Essentially equivalent would be the assumption that $H \Omega$ is the exterior derivative of some reasonable smooth $n$ form $\omega_{H}$ on $N$; then the $H$ volume could be defined by $\mathbf{V}_{H}\left(T, T_{0}\right)=\left\langle T-T_{0}, \omega_{H}\right\rangle$. In the context of parametric surfaces in 3 manifolds, such an approach was used recently by Toda [To].) With the condition $\int_{N} H d \mathcal{H}^{n+1}=0$ it is indeed straightforward to apply the reasoning of Section 3 to a closed manifold $N$. However, as we have mentioned in the Introduction we find it undesirable to exclude constant $H \not \equiv 0$ and we therefore consider an alternative way to circumvent the problem of ambiguity of the $H$ volume. Namely, we require that the current $Q$ in (4.1) has a support of mass less than $\frac{1}{2} \mathcal{H}^{n+1}(N)$. Such a current $Q$ is then unique, and the $H$ volume $\mathbf{V}_{H}\left(T, T_{0}\right)$ is well defined by (4.1) (if $Q$ exists).

For closed manifolds $N$ the isoperimetric inequalities for sets $A \subset N$ with finite perimeter take the following form:

$$
\begin{aligned}
& \min \left\{\mathcal{H}^{n+1}(A), \mathcal{H}^{n+1}(N \backslash A)\right\} \leq c \mathbf{M}(\partial \llbracket A \rrbracket), \\
& \min \left\{\mathcal{H}^{n+1}(A), \mathcal{H}^{n+1}(N \backslash A)\right\} \leq \gamma \mathbf{M}(\partial \llbracket A \rrbracket)^{(n+1) / n}
\end{aligned}
$$

and, more generally,

$$
b\left(\min \left\{\mathcal{H}^{n+1}(A), \mathcal{H}^{n+1}(N \backslash A)\right\}\right) \leq \mathbf{M}(\partial \llbracket A \rrbracket),
$$

where the (largest possible) function $b(t)$ is the isoperimetric profile of $N$. Note that, by Proposition 2.2, on each closed manifold $N$ an inequality (4.3) is valid with some finite constant $\gamma$ and implies (4.2) with $c=\left(\frac{1}{2} \gamma^{n} \mathcal{H}^{n+1}(N)\right)^{1 /(n+1)}$. For a Euclidean sphere $N_{\kappa}$ of curvature $\kappa^{2}>0$ the isoperimetric profile can be determined explicitely using the isoperimetric property of balls in $N_{\kappa}$. Equality holds in (4.4) if $A, N \backslash A$ are complementary spherical caps in $N_{\kappa}$. For general estimates of the isoperimetric constants and the function $b(t)$ in terms of geometrical data of $N$ we refer to Remark 2.4 (3). In particular, we recall the Lévy-Gromov inequality $[\mathrm{Gr}],[\mathrm{GHL}, 4 \mathrm{H}],[\mathrm{BZ}, 34.3]$ according to which the isoperimetric profile of a closed manifold $N$ with Ricci curvatures bounded 
from below by $n \kappa^{2}>0$ is not smaller $\alpha(\kappa, N)$ times the corresponding function of a sphere with $n+1$ volume equal to $\mathcal{H}^{n+1}(N)$, where $\alpha(\kappa, N)^{n+1}=$ $\kappa^{n+1} \mathcal{H}^{n+1}(N) \omega(n+1)^{-1}$ (and $\omega(n+1)$ the volume of the unit sphere in $\mathbb{R}^{n+2}$ ).

To see what the isoperimetric inequalities for sets of finite perimeter in $N$ imply for integer multiplicity currents $Q \in \mathcal{R}_{n+1}(N)$, we observe that the following abstract embedding theorem, anologous to (2.9), is valid for closed manifolds $N$ (we use the notation introduced in Section 2): For any $f \in B V(N)$ there exists $t_{0} \in \mathbb{R}$ such that the inequality

$$
\left|\int_{N}\left(f-t_{0}\right) h d \mathcal{H}^{n+1}\right| \leq\|\partial f\|(N),
$$

is true whenever the function $h \in L^{1}(A)$ satisfies

$$
\left|\int_{A} h d \mathcal{H}^{n+1}\right| \leq \mathbf{M}(\partial \llbracket A \rrbracket)
$$

for all sets $A \subset N$ of finite perimeter with $\mathcal{H}^{n+1}(A) \leq \frac{1}{2} \mathcal{H}^{n+1}(N)$. Indeed, since $\mathcal{H}^{n+1}([f>t])$ is a nonincreasing function of $t$ we determine $t_{0}$ such that $\mathcal{H}^{n+1}([f>t]) \leq \frac{1}{2} \mathcal{H}^{n+1}(N)$ holds for all $t>t_{0}$ and $\mathcal{H}^{n+1}([f \leq t]) \leq \frac{1}{2} \mathcal{H}^{n+1}(N)$ for all $t<t_{0}$. From Fubini's theorem we have

$$
\int\left(f-t_{0}\right) h d \mathcal{H}^{n+1}=\int_{t_{0}}^{\infty} \int_{[f>t]} h d \mathcal{H}^{n+1} d t-\int_{-\infty}^{t_{0}} \int_{[f \leq t]} h d \mathcal{H}^{n+1} d t,
$$

and with (4.6), the coarea formula and $\partial \llbracket f \leq t \rrbracket=-\partial \llbracket f>t \rrbracket$ we obtain the asserted inequality

$$
\left|\int_{N}\left(f-t_{0}\right) h d \mathcal{H}^{n+1}\right| \leq \int_{-\infty}^{\infty} \mathbf{M}(\partial \llbracket f>t \rrbracket) d t=\|\partial f\|(N) .
$$

We note that we can choose $t_{0} \in \mathbb{Z}$ if $f$ is integer valued, i.e. $f=\zeta_{Q}$ for some $Q \in \mathcal{R}_{n+1}(N)$. We also see that we can choose $t_{0}=0$ if $\operatorname{spt} f$ (or, at least, each of the sets spt $f_{+}$, spt $f_{-}$) has $\mathcal{H}^{n+1}$ measure $\leq \frac{1}{2} \mathcal{H}^{n+1}(N)$. If we know (4.6) only for $A$ with $\mathbf{M}(\partial \llbracket A \rrbracket) \leq s$, then we can still deduce (4.5) for $f$ with $\mathbf{M}(\partial \llbracket f>t \rrbracket) \leq s$ for $\mathcal{H}^{1}$ almost all $t$, e.g. for $f=\zeta_{Q}$ with $\mathbf{M}(Q) \leq s$.

If an isoperimetric inequality (4.2) holds, then (4.6) is satisfied for $h$ with $\sup |h| \leq c^{-1}$, hence (4.5) implies for $f \in B V(N)$

$$
\int_{N}\left|f-t_{0}\right| d \mathcal{H}^{n+1} \leq c\|\partial f\|(N) \quad \text { for some } t_{0} \in \mathbb{R}
$$

and for $Q \in \mathcal{R}_{n+1}(N)$

$$
\mathbf{M}\left(Q-t_{0} \llbracket N \rrbracket\right) \leq c \mathbf{M}(\partial Q) \quad \text { for some } t_{0} \in \mathbb{Z},
$$

where we have $t_{0}=0$ if $\mathcal{H}^{n+1}(\operatorname{spt} Q) \leq \frac{1}{2} \mathcal{H}^{n+1}(N)$. Of course, this is well known (e.g. [Ya]), and follows also immediately from (4.2) and the decomposition theorem. If, on the other hand, the "nonlinear" isoperimetric inequality 
(4.3) is valid, then Hölder's inequality gives (4.6) for all functions $h$ with $L^{n+1}$ norm bounded by $\gamma^{-n /(n+1)}$, hence (4.5) implies

$$
\int_{N}\left|f-t_{0}\right|^{(n+1) / n} d \mathcal{H}^{n+1} \leq \gamma(\|\partial f\|(N))^{(n+1) / n},
$$

and

$$
\int_{N}\left|\zeta_{Q}-t_{0}\right|^{(n+1) / n} d \mathcal{H}^{n+1} \leq \gamma \mathbf{M}(\partial Q)^{(n+1) / n} .
$$

If one knows more about the isoperimetric profile $b(t)$ of $N$, then one can derive further estimates for $f-t_{0}$ and $Q-t_{0} \llbracket N \rrbracket$ in terms of $\|\partial f\|(N)$ and $\mathbf{M}(\partial Q)$ respectively; see Section 2.

We can now repeat the reasoning from Section 3 with the following modification of definitions appropriate for closed manifolds. The $H$ volume $\mathbf{V}_{H}\left(T, T_{0}\right)$ for two homologous currents $T, T_{0} \in \mathcal{R}_{n}(N)$ is defined by

$$
\begin{aligned}
& \mathbf{V}_{H}\left(T, T_{0}\right)=\langle Q, H \Omega\rangle=\int_{N} \zeta_{Q} H d \mathcal{H}^{n+1} \\
& \text { with } Q \in \mathcal{R}_{n+1}(N), \partial Q=T-T_{0},
\end{aligned}
$$

provided we have $\int_{N} H d \mathcal{H}^{n+1}=0$ (so that the choice of $Q$ does not matter) or $Q$ can be chosen with $\mathcal{H}^{n+1}(\operatorname{spt} Q)<\frac{1}{2} \mathcal{H}^{n+1}(N)$ (so that, with this extra condition, $Q$ is unique). By an isoperimetric condition of type $(c, s)$ for $H$ on $N$ we now mean an inequality

$$
\left|\int_{A} H d \mathcal{H}^{n+1}\right| \leq c \mathbf{M}(\partial \llbracket A \rrbracket)
$$

valid for all sets of finite perimeter $A \subset N$ with $\mathbf{M}(\partial \llbracket A \rrbracket) \leq s$ and $\mathcal{H}^{n+1}(A) \leq$ $\frac{1}{2} \mathcal{H}^{n+1}(N)$. The isoperimetric constant $c_{N}(s)$ of $N$ is defined for $0 \leq s \leq \infty$ as the smallest constant $c$ such that (4.2) is valid for all sets of finite perimeter $A \subset N$ with $\mathbf{M}(\partial \llbracket A \rrbracket) \leq s$. Note that (4.11), (4.5), (4.6) and (4.12) imply the volume estimate

$$
\left|\mathbf{V}_{H}\left(T, T_{0}\right)\right| \leq c \mathbf{M}\left(T-T_{0}\right),
$$

whenever an isoperimetric condition of type $(c, s)$ holds for $H, \mathbf{M}\left(T-T_{0}\right) \leq s$, and the $H$ volume $\mathbf{V}_{H}\left(T, T_{0}\right)$ is defined (so that $\int_{N} H d \mathcal{H}^{n+1}=0$ or we can choose $t_{0}=0$ for $f=\zeta_{Q}$ in (4.5), in any case $\left.\int_{N} t_{0} H d \mathcal{H}^{n+1}=0\right)$.

4.1. Theorem. Suppose $\int_{N} H d \mathcal{H}^{n+1}=0$ and $H$ satisfies an isoperimetric condition of type $(c, \infty)$ on $N$ with $c<1$. Then for any $T_{0} \in \mathcal{R}_{n}(N)$ there exists a current $T \in \mathcal{R}_{n}(N)$ with prescribed mean curvature $H$ and homologous to $T_{0}$ in $N\left(\right.$ in particular $\left.\partial T=\partial T_{0}\right)$.

Since we obtain the current $T$ by minimizing $\mathbf{E}_{H}$ on the class $\mathcal{C}\left(T_{0}, N\right)$ as in Section 3 (cf. Definition 3.1 (i)), $T$ will have all the properties stated in Remark 3.3 . 
Proof. $\quad \mathbf{E}_{H}(T)=\mathbf{M}(T)+\mathbf{V}_{H}\left(T, T_{0}\right)$ is well defined on $\mathcal{C}\left(T_{0}, N\right)$, since $\int_{N} H d \mathcal{H}^{n+1}=0$. As in the proof of Theorem 3.2 we see from (4.13) and $c<1$ that minimizing sequences $T_{k}$ for $\mathbf{E}_{H}$ on $\mathcal{C}\left(T_{0}, N\right)$ are mass bounded. From (4.8) it follows that the currents $Q_{k} \in \mathcal{R}_{n+1}(N)$ with $\partial Q_{k}=T_{k}-T_{0}$ can be chosen to satisfy $\mathbf{M}\left(Q_{k}\right) \leq c \mathbf{M}\left(T_{k}-T_{0}\right)$ so that the $Q_{k}$ are bounded in mass as well (even the $L^{n+1}$ norm of the multiplicity functions $\zeta_{Q_{k}}$ is bounded, by (4.10)). Hence, the $B V$ compactness theorem implies, for a subsequence, weak convergence $T_{k} \rightarrow T \in \mathcal{R}_{n}(N)$ and $L^{1}$ convergence $\zeta_{Q_{k}} \rightarrow \zeta_{Q}$, where $Q \in \mathcal{R}_{n+1}(N)$ satisfies $\partial Q=T-T_{0}$. Convergence of the volumes $\mathbf{V}_{H}\left(T_{k}, T_{0}\right) \rightarrow \mathbf{V}_{H}\left(T, T_{0}\right)$ then follows from (4.11), $T$ is $\mathbf{E}_{H}$ minimizing in $\mathcal{C}\left(T_{0}, N\right)$ by lower semicontinuity of mass, and $T$ has prescribed mean curvature $H$ by Lemma 1.3 .

The sufficient criteria for isoperimetric conditions from Proposition 3.4 also apply to the present case of a closed manifold $N=G$. Moreover, it is now sufficient to consider sets $A$ of finite perimeter with $\mathcal{H}^{n+1}(A) \leq \frac{1}{2} \mathcal{H}^{n+1}(N)$. As an example we state as a corollary what follows from the criteria (i) and (ii) in Proposition 3.4. It is clear that, as in Section 3, one can also formulate existence results for currents of prescribed mean curvature in closed manifold which are based on the remaining criteria stated in Proposition 3.4. We also note that in part (ii) of the corollary it would be sufficient to have the hypothesis for $H_{+}$, $H_{-}$separately, instead of $|H|=H_{+}+H_{-}$.

4.2. Corollary. The conclusion of Theorem 4.1 is true if, in addition to $\int_{N} H d \mathcal{H}^{n+1}=0$, one of the following conditions holds:

(i) $\sup _{N}|H|<c^{-1}$, where $c$ is the constant from the isoperimetric inequality (4.2) (valid for all $A$ );

(ii) $\int_{N}|H|^{n+1} d \mathcal{H}^{n+1}<\gamma^{-n}$, where $\gamma$ is the constant from the isoperimetric inequality (4.3) (valid for all $A$ ).

We now turn to the existence results in a closed manifold which do not assume the condition $\int_{N} H d \mathcal{H}^{n+1}=0$ for the prescribed mean curvature. Since we then require $\mathcal{H}^{n+1}(\operatorname{spt} Q)<\frac{1}{2} \mathcal{H}^{n+1}(N)$ for currents $Q \in \mathcal{R}_{n+1}(N)$, in order to define the volume $\mathbf{V}_{H}\left(T, T_{0}\right)$ for $T, T_{0} \in \mathcal{R}_{n}(N)$ with $T-T_{0}=\partial Q$ uniquely, we have to work with a mass restriction $\mathbf{M}\left(T-T_{0}\right) \leq s$, which implies $\mathbf{M}(Q)<$ $\frac{1}{2} \mathcal{H}^{n+1}(N)$ for a suitably chosen current $Q$ via the inequality (4.8). Otherwise we proceed exactly as in Theorem 3.11 .

4.3. Theorem. Suppose $0<s<\infty$, the isoperimetric constant $c_{N}(s)$ satisfies $s c_{N}(s)<\frac{1}{2} \mathcal{H}^{n+1}(N)$, and $T_{0} \in \mathcal{R}_{n}(N)$ with $2 \mathbf{M}\left(T_{0}\right)<s$. Then there exists a current $T \in \mathcal{R}_{n}(N)$ with prescribed mean curvature $H$ and homologous to $T_{0}$ in $N$, provided

$$
\sup _{N}|H|<\frac{s-2 \mathbf{M}\left(T_{0}\right)}{s c_{N}(s)} .
$$


Proof. We want to minimize $\mathbf{E}_{H}$ on $\mathcal{C}\left(T_{0}, N, \sigma\right)$ (cf. Definition 3.1 (i)) with $\sigma>1,(1+\sigma) \mathbf{M}\left(T_{0}\right) \leq s$; the best choice is $\sigma=s \mathbf{M}\left(T_{0}\right)^{-1}-1$. Note that we have

$$
\mathbf{M}(\partial Q)=\mathbf{M}\left(T-T_{0}\right) \leq \mathbf{M}\left(T_{0}\right)+\mathbf{M}(T) \leq(1+\sigma) \mathbf{M}\left(T_{0}\right) \leq s
$$

whenever $T=T_{0}+\partial Q \in \mathcal{C}\left(T_{0}, N, \sigma\right)$, and observe that (4.8) holds with $c$ replaced by $c_{N}(s)$ when $\mathbf{M}(\partial Q) \leq s$. Therfore, the hypothesis $s_{N}(s)<$ $\frac{1}{2} \mathcal{H}^{n+1}(N)$ and (4.8) imply that $Q$ can be chosen to satisfy

$$
\mathbf{M}(Q) \leq c_{N}(s) \mathbf{M}(\partial Q) \leq c_{N}(s) s<\frac{1}{2} \mathcal{H}^{n+1}(N) .
$$

It follows that the $H$ volume and the $H$ energy are well defined on $\mathcal{C}\left(T_{0}, N, \sigma\right)$. We can now proceed exactly as in the proof of Theorem 3.11 to find a minimizer $T$ in $\mathcal{C}\left(T_{0}, N, \sigma\right)$ and prove $\mathbf{M}(T)<\sigma \mathbf{M}\left(T_{0}\right)$ if (4.14) holds (cf. (3.12)). Note that (4.15) for the currents $Q_{k}$ associated with a minimizing sequence $T_{k}$ implies the same inequality also for the limit $Q$ (of a subsequence) so that $\lim _{k \rightarrow \infty} \mathbf{V}_{H}\left(T_{k}, T_{0}\right)=\mathbf{V}_{H}\left(T, T_{0}\right)$ follows for $T=T_{0}+\partial Q$ from (4.14) and the definition of the $H$ volume in the present situation.

Instead of (4.14) we could again use other conditions for $H$ that ensure the validity of an isoperimetric condition of type $(c, s)$ with a constant $c$ satisfying $s-2 \mathbf{M}\left(T_{0}\right)>s c$. It is instructive to apply the theorem to the standard sphere $N=S^{n+1}$ :

4.4. Example. For $N=S^{n+1}$ we have (with the functions $\alpha_{\kappa}, \beta_{\kappa}$ introduced in Example 2.5, here $\kappa=1$ )

$$
s c_{N}(s)=\alpha_{\kappa} \circ \beta_{\kappa}^{-1}(s) \text { for } 0 \leq s \leq \mathcal{H}^{n}\left(S^{n}\right)=\omega(n)
$$

and, in particular,

$$
s c_{N}(s) \begin{cases}=\frac{1}{2} \omega(n+1)=\frac{1}{2} \mathcal{H}^{n+1}(N) & \text { for } s=\omega(n), \\ <\frac{1}{2} \mathcal{H}^{n+1}(N) & \text { for } 0 \leq s<\omega(n) .\end{cases}
$$

The hypothesis $s c_{N}(s)<\frac{1}{2} \mathcal{H}^{n+1}(N)$ in Theorem 4.3 is therefore equivalent to $s<\omega(n)$, and $2 \mathbf{M}\left(T_{0}\right)<s$ means that the mass of $T_{0}$ should be smaller than half the mass of a great $n$ sphere in $S^{n+1}$. If this is satisfied, then we can find, for sufficiently small prescribed $H \not \equiv 0$, a current $T \in \mathcal{R}_{n}\left(S^{n+1}\right)$ with prescribed mean curvature $H$ and boundary $\partial T=\partial T_{0}$. In the limiting case $2 \mathbf{M}\left(T_{0}\right)=\omega(n)$ the theorem does not apply, and indeed, no $n$ current with constant mean curvature $H \not \equiv 0$ and boundary $\partial T_{0}$ does exist when $T_{0}$ represents an oriented half great $n$ sphere in $S^{n+1}$ (cf. Remark 1.4).

In order to optimize the restriction (4.14) on $\sup |H|$ for a given $T_{0}$ with $2 \mathrm{M}\left(T_{0}\right)<\omega(n)$ we have to determine the maximum of the function

$$
\frac{s-2 \mathbf{M}\left(T_{0}\right)}{s c_{N}(s)}=\frac{\beta_{\kappa}(\varrho)-2 \mathbf{M}\left(T_{0}\right)}{\alpha_{\kappa}(\varrho)} \text { for } s=\beta_{\kappa}(\varrho)
$$


on the intervall $\beta_{\kappa}^{-1}\left(2 \mathbf{M}\left(T_{0}\right)\right)<\varrho<\pi / 2$. Using the computations from Example 2.5, one checks that the derivative equals $\alpha_{\kappa}(\varrho)^{-2} \beta_{\kappa}(\varrho) \eta(\varrho)$, where $\eta(\varrho)=\alpha_{\kappa}(r) n \cot \varrho-\beta_{\kappa}(\varrho)+2 \mathbf{M}\left(T_{0}\right)$ is strictly decreasing with exactly one zero in the interval considered. Therefore, a unique maximum point $r$ exists in the interval, and then

$$
\sup |H|<n \cot r
$$

is the weakest possible restriction on $H$ allowed by Theorem 4.3. With the inequalities for $c_{\kappa}(r)$ from Example 2.5 one verifies further

$$
\alpha_{\kappa}(\varrho) n \cot \varrho-\beta_{\kappa}(\varrho)+n \alpha(n+1) \int_{0}^{\varrho} \sin ^{n-1} \tau d \tau>0
$$

(the function is increasing with limit 0 as $\varrho \downarrow 0$ ). This implies

$$
2 \mathbf{M}\left(T_{0}\right)>n \alpha(n+1) \int_{0}^{\varrho} \sin ^{n-1} \tau d \tau,
$$

which may be formulated in geometric terms by saying that $r$ is smaller than the radius $R$ of a ball in $S^{n+1}$ that posesses an equatorial $n$ disc of $\mathcal{H}^{n}$ measure $2 \alpha(n) \mathbf{M}\left(T_{0}\right) / \alpha(n+1)$. The condition $\sup |H|<n \cot R$ is hence sufficient for existence of a current with mean curvature $H$ and boundary $\partial T_{0}$ in $S^{n+1}$. Taking the limit $s \uparrow \omega(n)$, we see that the more explicit condition

$$
\sup |H|<2 \frac{\omega(n)-2 \mathbf{M}\left(T_{0}\right)}{\omega(n+1)}
$$

is also sufficient for existence. This condition is, however, much more restrictive than necessary (for small $\mathbf{M}\left(T_{0}\right)$, in particular). But also the weaker condition $\sup |H|<n \cot r$ above may be too restrictive. This is illustrated by $T_{0}$ representing a half $n$ sphere of radius $\varrho$ in $S^{n+1}$. Then, we know from Theorem 3.10 the existence for mean curvatures $H$ not exceeding in absolute value the mean curvature $n \cot \varrho$ of this half sphere; such a result is optimal by Remark 1.4. It does not follow from the existence criteria above, however, since $r$ is larger than $\varrho$.

By the preceding example, Theorem 4.3 is optimal with regard to the conditions imposed on $T_{0}$, whereas it seems very unlikely that optimality holds also for the condition (4.14) on the size of the allowed mean curvature functions. Exactly as in Corollary 3.13, it can be seen, however, that (4.14) allows large mean curvature functions $H$ if only the mass of $T_{0}$ is small. We formulate this more precisely, noting that $s_{0} c_{N}\left(s_{0}\right)<\frac{1}{2} \mathcal{H}^{n+1}(N)$ is always true for sufficiently small $s_{0}>0$, by Proposition 2.2:

4.5. Corollary. Suppose $s_{0} c_{N}\left(s_{0}\right)<\frac{1}{2} \mathcal{H}^{n+1}(N)$, the isoperimetric inequality (4.3) is valid with the constant $\gamma$ for all $A \subset N$ with $\mathbf{M}(\partial \llbracket A \rrbracket) \leq s_{0}$, 
and $T_{0} \in \mathcal{R}_{n}(N)$ with $2(n+1) \mathbf{M}\left(T_{0}\right) \leq s_{0}$. Then there exists a current $T$ of prescribed mean curvature $H$ and homologous to $T_{0}$, whenever

$$
\sup _{N}|H|<\frac{n}{(n+1) \gamma\left[2(n+1) \mathbf{M}\left(T_{0}\right)\right]^{1 / n}} .
$$

We conclude with an observation concerning the proof of Theorem 4.3. There we have applied the isoperimetric inequality $\mathbf{M}(Q) \leq c \mathbf{M}\left(T-T_{0}\right)$ for $Q \in$ $\mathcal{R}_{n+1}(N)$ with $\partial Q=T-T_{0}$, but in the proof we only used the weaker inequality

$$
\mathbf{M}(Q) \leq c\left(\mathbf{M}(T)+\mathbf{M}\left(T_{0}\right)\right) .
$$

If we can find $c$ and $\sigma>1$ such that (4.16) holds for all $T \in \mathcal{C}\left(T_{0}, N, \sigma\right)$ (i.e. $T \in \mathcal{R}_{n}(N)$ homologous to $T_{0}$ with $\mathbf{M}(T) \leq \sigma \mathbf{M}\left(T_{0}\right)$, see Definition 3.1) with some $Q \in \mathcal{R}_{n+1}(N)$ satisfying $\partial Q=T-T_{0}$, and if also

$$
c(1+\sigma) \mathbf{M}\left(T_{0}\right)<\frac{1}{2} \mathcal{H}^{n+1}(N)
$$

holds, then the proof of the theorem remains valid and produces a current $T \in \mathcal{R}_{n}(N)$ with prescribed mean curvature $H$ homologous to $T_{0}$, provided

$$
c \sup _{N}|H| \leq \frac{\sigma-1}{\sigma+1} .
$$

To fulfill (4.16) we choose, for a given $0 \neq T_{0} \in \mathcal{R}_{n}(N)$,

$$
\begin{aligned}
c=\sup \left\{\frac{\mathbf{M}(Q)}{\mathbf{M}\left(T_{0}\right)+\mathbf{M}(T)}: Q \in \mathcal{R}_{n+1}(N),\right. & \\
\mathbf{M}(Q) & \left.\leq \frac{1}{2} \mathcal{H}^{n+1}(N), T=T_{0}+\partial Q\right\},
\end{aligned}
$$

and observe that this supremum is attained (by the $B V$ compactness theorem) for some $Q$ and $T=T_{0}+\partial Q$. If we have $c=\frac{1}{4} \mathcal{H}^{n+1}(N) / \mathbf{M}\left(T_{0}\right)$, then $\mathbf{M}(T)=$ $\mathbf{M}\left(T_{0}\right)$ and $\mathbf{M}(Q)=\frac{1}{2} \mathcal{H}^{n+1}(N)$ must hold. This is impossible if $T_{0}$ is the unique mass minimizer in its homology class or if no other minimizer exists in this homology class that, together with $T_{0}$, bounds an $n+1$ current of mass exactly $\frac{1}{2} \mathcal{H}^{n+1}(N)$. Assuming this, we have $c<\frac{1}{4} \mathcal{H}^{n+1}(N) / \mathbf{M}\left(T_{0}\right)$ and we can choose $\sigma>1$ such that (4.17) is satisfied. We therefore have proved the following result:

4.6. Theorem. Suppose $N$ is a closed manifold, $0 \neq T_{0} \in \mathcal{R}_{n}(N)$ is mass minimizing in its homology class, and no other mass minimizer $T_{1}$ exists in this class such that $T_{1}-T_{0}$ bounds an $n+1$ current of mass $\frac{1}{2} \mathcal{H}^{n+1}(N)\left(\right.$ e.g. $T_{0}$ is the unique minimizer). Then there exists $\delta>0$ such that for each prescribed mean curvature function $H$ with $\sup _{N}|H| \leq \delta$ we can find a current $T \in \mathcal{R}_{n}(N)$ of prescribed mean curvature $H$ and homologous to $T_{0}$ in $N$. 
It is instructive to consider a torus $N=\left(S^{1}\right)^{n+1}$ or, more generally, $N=$ $M \times S^{1}$ as an example. If we choose the product metric on $N$ and $T_{0}=$ $\llbracket M \times\left\{e^{i \theta}\right\} \rrbracket$, then $T_{0}$ does not satisfy the hypotheses of the theorem (consider $T_{1}=\llbracket M \times\left\{e^{i \theta+\pi}\right\} \rrbracket$ as another minimizer), and indeed, no current with nonvanishing constant mean curvature and homologous to $T_{0}$ exists in $N$, by Remark 1.4. On the other hand, we can perturb the product metric to make $T_{0}$ the unique minimizer in its homology class, and then Theorem 4.6 applies. Another example is $N=S^{n+1}$ and $T_{0}$ a great half $n$ sphere in $S^{n+1}$.

Thinking of cylinders $M \times \mathbb{R}$, one would expect that a similar discussion should also apply to non-closed manifolds $N$. The only problem with the argumentation above is then that the supremum (4.19) may not be attained, if the supports of the currents $Q$ are not restricted to a fixed compact set. Instead of the mass bound $\mathbf{M}(Q) \leq \frac{1}{2} \mathcal{H}^{n+1}(N)$ we now use in (4.19) the condition $\mathbf{M}(Q) \leq t$ with some parameter $t<\infty$, i.e. we consider, for $0 \neq T_{0} \in \mathcal{R}_{n}(N)$ homologically mass minimizing,

$$
\begin{aligned}
c=\sup \left\{\frac{\mathbf{M}(Q)}{\mathbf{M}\left(T_{0}\right)+\mathbf{M}(T)}: Q \in \mathcal{R}_{n+1}(N),\right. & \\
& \left.\mathbf{M}(Q) \leq t, T=T_{0}+\partial Q\right\},
\end{aligned}
$$

and we want to argue that $c<\frac{1}{2} t / \mathbf{M}\left(T_{0}\right)$. Otherwise the supremum could only be attained when $T$ is mass minimizing in the homology class of $T_{0}$ and $\mathbf{M}(Q)=t$. In any case, if $c \geq \frac{1}{2} t / \mathbf{M}\left(T_{0}\right)$, then for a minimizing sequence $T_{k}, Q_{k}$ we must have $\mathbf{M}\left(T_{k}\right) \rightarrow \mathbf{M}\left(T_{0}\right)$ and $\mathbf{M}\left(Q_{k}\right) \rightarrow t$. Passing to convergent subsequences, we get $\zeta_{Q_{k}} \rightarrow \zeta_{Q}$ in $L_{\text {loc }}^{1}(N)$ and $T_{k} \rightarrow T=T_{0}+\partial Q$ weakly. Since $T_{0}$ is mass minimizing, $\mathbf{M}(T)=\mathbf{M}\left(T_{0}\right)$ follows. This implies that $\mathbf{M}\left(T_{k}\llcorner(N \backslash B))\right.$ is uniformly small for large balls $B \subset N$, and with slicing as in the proof of Theorem 3.2 we see, using also homogeneous regularity of $N$ and Proposition 2.2, that $\mathbf{M}\left(Q_{k}\llcorner(N \backslash B))\right.$ is uniformly small, too. We conclude $\mathbf{M}(Q)=\lim _{k \rightarrow \infty} \mathbf{M}\left(Q_{k}\right)=t$, and the supremum (4.20) is attained. The result of all these considerations is that $c<\frac{1}{2} t / \mathbf{M}\left(T_{0}\right)$ must hold, if no mass minimizer $T_{1}$ exists in the homology class of $T_{0}$ such that $T_{1}-T_{0}$ bounds an $n+1$ current of mass $t$. We can then chose $\sigma>1$ with $c(1+\sigma) \mathbf{M}\left(T_{0}\right) \leq t$ and proceed exactly as before to deduce the following result:

4.7. Theorem. Suppose $N$ is non-closed as in Section 3, in particular non-compact and homogeneously regular. Suppose further that $0 \neq T_{0} \in \mathcal{R}_{n}(N)$ is mass minimizing in its homology class and that there exists $0<t<\infty$ such that no other mass minimizer $T_{1}$ bounds together with $T_{0}$ an $n+1$ current of mass $t$ in $N$. Then for all prescribed mean curvature functions $H$ with $\sup |H|$ sufficiently small there exists $T \in \mathcal{R}_{n}(N)$ with mean curvature $H$ and homologous to $T_{0}$ in $N$.

The hypothesis for $T_{0}$ is satisfied when $T_{0}$ is the unique mass minimizer in its homology class, or when all the minimizers have support in a fixed compact subset of $N$. 


\section{REFERENCES}

[Ad] R. A. Adams: Sobolev spaces. Academic Press, New York San Francisco London, 1975.

[Al] W. K. Allard: On the first variation of a varifold. Ann. Math. 95 (1972), 417-491.

[Au1] T. Aubin: Problèmes isopérimétriques et espaces de Sobolev. J. Differential Geometry 11 (1976), 573-598.

[Au2] T. Aubin: Non-linear analysis on manifolds, Monge-Ampère equations. Springer-Verlag, New York Heidelberg Berlin, 1982.

[BZ] Y. D. Burago And V. A. Zalgaller: Geometric inequalities. SpringerVerlag, New York Heidelberg Berlin, 1988.

[Ch] J. CheEger: A lower bound for the smallest eigenvalue of the Laplacian. pp 195-199 in "Problems in Analysis; a Symposium in Honor of S. Bochner". Princeton University Press, Princeton, 1970.

[Cr1] C. B. Croke: Some isoperimetric inequalities and eigenvalue estimates. Ann. Sci. Éc. Norm. Sup. 13 (1980), 419-435.

[Cr2] C. B. CROKE: A sharp four dimensional isoperimetric inequality. Comment. Math. Helvetici 59 (1984), 187-192.

[CS] T. Coulhon, L. SAlofF-Coste: Isopérimétrie pour les groupes et les variétés. Rev. Mat. Iberoamericana 9 (1993), 293-314.

[DeG] E. De Giongi: Sulla proprietà isoperimetrica dell' ipersfera, nelle classe degli insiemi avanti frontiera orientata di misura finita. Atti. Accad. Naz. Lincei, ser 1, 5 (1958), 33-44.

[Du] F. DuZAAR: On the existence of surfaces with prescribed mean curvature and boundary in higher dimensions. Anal. non lin. 10 (1993), 191-214.

[DF1] F. DuZAar, M. Fuchs: On the existence of integral currents with prescribed mean curvature vector. Manuscripta Math. 67 (1990), 41-67.

[DF2] F. DuZAAR, M. Fuchs: A general existence theorem for integral currents with prescribed mean curvature form. Bolletino U.M.I. (7) 6-B (1992), 901-912.

[DS1] F. DuzaAR, K. Steffen: $\lambda$ minimizing currents. Manuscripta Math. 80 (1993), 403-447.

[DS2] F. DuZAAR, K. StefFen: Boundary regularity for minimizing currents with prescribed mean curvature. Calc. Var. 1 (1993), 355-406.

[DS3] F. DuzaAR, K. Steffen: Comparison principles for hypersurfaces of prescribed mean curvature. J. reine angew. Math. 457 (1994), 71-83.

[DS4] F. Duzana, K. Steffen: Parametric surfaces of least H-energy in a Riemannian manifold. In preparation.

[EG] L. C. Evans, L. F. GariePy: Measure theory and fine properties of functions. CRC Press, Boca Raton Ann Arbor London, 1992.

[Fe] H. Federer: Geometric Measure Theory. Springer-Verlag, Berlin Heidelberg New York, 1969.

[Ga] S. GALlot: Inegalités isopérimétriques et analytiques sur les variétés Riemanniennes. pp 31-91 in Astérique, vols. 163-164, Soc. Math. France, Paris, 1988.

[Gr] M. Gromov: Paul Lévy's isoperimetric inequality. IHES/M/80/320, Inst. Hautes Étud. Sci., Bures-sur-Yvette, 1980.

[Gü] P. GüNTHER: Einige Vergleichssätze über das Volumenelement eines Riemannschen Raumens. Publ. Math. Debrecen 7 (1960), 258-287. 
[Gu1] R. Gulliver: The Plateau problem for surfaces of prescribed mean curvature in a Riemannian manifold. J. Differential Geometry 8 (1972), 317-339.

[Gu2] R. Gulliver: On the non-existence of a hypersurface of prescribed mean curvature with a given boundary. Manuscripta Math. 11 (1974),15-39.

[Gu3] R. Gulliver: Necessary conditions for submanifolds and currents with prescribed mean curvature vector. Seminar on Minimal Submanifolds, ed. E. Bombieri, Princeton, 1983.

[GHL] S. Gallot, D. Hulin and J. Lafontaine: Riemannian Geometry. $2^{\text {nd }}$ edition. Springer-Verlag, Berlin Heidelberg New York, 1990.

[GS1] R. Gulliver AND J. SpRUCK: The Plateau problem for surfaces of prescribed mean curvature in a cylinder. Invent. Math. 13 (1971), 169-178.

[GS2] R. Gulliver And J. SpRuck: Existence theorems for parameric surfaces of prescribed mean curvature. Indiana Univ. Math. J. 22, (1972), 445-472.

[He1] E. HEINZ: Über die Existenz einer Fläche konstanter mittlerer Krümmung mit gegebener Berandung. Math. Ann. 127 (1954), 258-287.

[He2] E. HEINZ: On the non-existence of a surface of constant mean curvature with finite area and prescribed rectifiable boundary. Arch. Rat. Mech. Anal. 35 (1969), 249-252.

[Hi1] S. HildebrandT: On the Plateau problem for surfaces of prescribed mean curvature. Comm. Pure Appl. Math. 23 (1970), 97-114.

[Hi2] S. HildeBRANDT: Randwertprobleme für Flächen mit vorgeschriebener mittlerer Krümmung und Anwendungen auf die Kapillaritätstheorie I. Math. Z. 112 (1969), 205-213.

[Hi3] S. HildebrandT: Maximum principles for minimal surfaces and for surfaces of continuous mean curvature. Math. Z. 128 (1972), 253-269.

[Hi4] S. HiLdEBRANDT: On the regularity of solutions of two-dimensional variational problems with obstructions. Comm. Pure Appl. Math. 25 (1972), 479496.

[HeK] E. Heintze And H. KARChER: A general comparison theorem with applications to volume estimates for submanifolds. Ann. Sci. Éc. Norm. Sup. 11 (1978), 451-470.

[HiK] S. Hildebrandt AND H. KAUL: Two-dimensional variational problems with obstructions, and Plateau's problem for $H$-surfaces in a Riemannian manifold. Comm. Pure Appl. Math. 25 (1972), 187-223.

[HSi] R. HARDT AND L. SimON: Boundary regularity and embedded solutions for the oriented Plateau problem. Ann. Math. 110 (1979), 439-486.

[HSp] D. Hoffman AND J. Spruck: Sobolev and isoperimetric inequalities for Riemannian submanifolds. Comm. Pure Appl. Math. 27 (1974), 715-727; correction ibid. 28 (1975), 765-766.

[Kl] B. KLEINER: An isoperimetric comparison theorem. Invent. math. 108 (1992), 37-47.

[Ma] V. G. MAZ'JA: Sobolev Spaces. Springer-Verlag, Berlin Heidelberg New York, 1985.

[MS] J. Michael And L. Simon: Sobolev and mean-value inequalities on generalized submanifolds of $\mathbb{R}^{n}$. Comm. Pure Appl. Math. 26 (1973), 361-379.

[Os] R. Ossermann: The isoperimetric inequality. Bull. Amer. Math. Soc. 87 (1978), 1182-1238.

[Sch] E. ScHMIDT: Beweis der isoperimetrischen Eigenschaft der Kugel im hyperbolischen und sphärischen Raum jeder Dimensionszahl. Math. Z. 49 (1943/44), 1-109. 
[Se] J. SERRIN: The problem of Dirichlet for quasilinear elliptic differential equations in many independent variables. Phil. Trans. Royal Soc. London 264 (1969), 413-419.

[Si] L. Simon: Lectures on geometric measure theory. Proc. Cent. Math. Anal. Austr. Nat. Univ., Vol. 3, Canberra, 1983.

[St1] K. Steffen: Isoperimetric inequalities and the problem of Plateau. Math. Ann. 222 (1976), 97-144.

[St2] K. StEFFEn: On the existence of surfaces with prescribed mean curvature and boundary. Math. Z. 146 (1976), 113-135.

[Str] M. Struwe: Plateau's problem and the calculus of variations. Mathematical Notes 35, Princeton University Press, Princeton, New Jersey, 1988.

[To] M. TodA: On the existence of $H$-surfaces into Riemannian manifolds. Calc. Var. 5 (1997), 55-83.

[We] H. C. WENTE: An existence theorem for surfaces of constant mean curvature. J. Math. Analysis Appl. 26 (1969), 249-252.

[Ya] S. T. YAU: Isoperimetric constants and the first eigenvalue of a compact Riemannian manifold. Ann. Sci. Éc. Norm. Sup., 83(1975), 487-507.

[Zi] W. P. Ziemer: Weakly differentiable functions. Springer-Verlag, New York Berlin Heidelberg, 1989.

Frank DUZAAR

Institut für Mathematik

Humboldt Universität zu Berlin

(Sitz: Ziegelstr. 13a), Unter den Linden 6

D-10099 Berlin Germany

E-MAIL: duzaar@mathematik.hu-berlin.de

Klaus StefFen

Mathematisches Institut der Heinrich-Heine-Universität Düsseldorf

Universitätstr. 1

D-40225 Düsseldorf GERMANY

E-MAIL: steffen@cs.uni-duesseldorf.de

Received: September 25th, 1995; revised: November 12th, 1996. 